\title{
1 ProtAnno, an Automated Cell Type Annotation Tool for Single \\ Cell Proteomics Data that integrates information from Multiple Reference Sources
}

Wenxuan Deng1, Biqing Zhu1, Seyoung Park2, Tomokazu S. Sumida3,4, Avraham

$8{ }^{1}$ Department of Biostatistics, Yale School of Public Health, New Haven, CT, USA

$9 \quad 2$ Department of Statistics, Sungkyunkwan University, South Korea

$10{ }^{3}$ Department of Neurology, Yale School of Medicine, New Haven, CT, USA

$11{ }^{4}$ Department of Immunobiology, Yale School of Medicine, New Haven, CT, USA

$12{ }^{5}$ Section of Pulmonary, Critical Care and Sleep Medicine Section, Department of Internal

13 Medicine, School of Medicine, Yale University, New Haven, CT, USA

$14{ }^{6}$ Program of Computational Biology and Bioinformatics, Yale University, New Haven, CT, 15 USA 


\section{Abstract}

26 Compared with sequencing-based global genomic profiling, cytometry labels targeted

27 surface markers on millions of cells in parallel either by conjugated rare earth metal

28 particles or Unique Molecular Identifier (UMI) barcodes. Correct annotation of these cells

29 to specific cell types is a key step in the analysis of these data. However, there is no

30 computational tool that automatically annotates single cell proteomics data for cell type

31 inference. In this manuscript, we propose an automated single cell proteomics data

32 annotation approach called ProtAnno to facilitate cell type assignments without laborious

33 manual gating. ProtAnno is designed to incorporate information from annotated single cell

34 RNA-seq (scRNA-seq), CITE-seq, and prior data knowledge (which can be imprecise) on

35 biomarkers for different cell types. We have performed extensive simulations to

36 demonstrate the accuracy and robustness of ProtAnno. For several single cell proteomics

37 datasets that have been manually labeled, ProtAnno was able to correctly label most single

38 cells. In summary, ProtAnno offers an accurate and robust tool to automate cell type

39 annotations for large single cell proteomics datasets, and the analysis of such annotated cell

40 types can offer valuable biological insights.

41 Keywords: CyTOF; scRNA-seq; CITE-seq; Gating; Data Integration. 


\section{Introduction}

50 Recent years have seen the developments of many single cell platforms(Eberwine et al.

51 2014) that have enabled researchers to collect high throughput -omics profiles at the

52 individual cell level, including genomics(Dong et al. 2017; Nagano et al. 2013) ,

53 transcriptomics(Hwang, Lee, and Bang 2018), proteomics(Labib and Kelley 2020), and

54 epigenomics(Fang et al. 2021). These data can reveal biological heterogeneity across

55 different biological conditions. They offer a direct approach to studying cell type

56 compositions and functional cell states. The most well-developed platforms are scRNA-seq

57 for transcriptomics and flow cytometry (CyTOF)(Bandura et al. 2009; Spitzer and Nolan

58 2016; Ornatsky et al. 2010) for proteomics. Notably, the rise of scRNA-seq has generated

59 rich data and resources on single cell transcriptomics(Regev et al. 2017; Lindeboom, Regev,

60 and Teichmann 2021).

61 In addition to collecting single cell data for one specific -oimcs data type, it is possible to 62 collect multi-omics data simultaneously at the single cell level, e.g., CITE-seq(Stoeckius et

63 al. 2017) that measures mRNA and antibody counts simultaneously by UMI barcode. These

64 data can characterize the cellular relationship between transcript and cell surface marker

65 abundance. Methods have been developed to bridge these two types of omics data. For

66 example, cTP-net(Zhou et al. 2020) is a transfer learning approach under a deep learning

67 framework to predict surface protein levels from scRNA-seq data. The generation of

68 diverse types of single cell data poses many computational challenges due to their high

69 dimensionality and large sample sizes. In this paper, we focus on the annotation of single

70 cell proteomics data to their corresponding cell types, which is a critical step in single cell

71 analysis.

72 A major advantage of single cell proteomics data compared with scRNA-seq data is their

73 high sensitivity and specificity. Based on surface marker expression patterns, manual

74 gating can be used to identify different cell populations by the expression distributions.

75 However, cell type labeling by manual gating on single cell data is labor intensive and

76 subjective as it highly depends on the expert who annotates the cells. Although some

77 methods can analyze these data based on state-of-the-art machine learning methods(Li et

78 al. 2017; Van Gassen et al. 2015; Levine et al. 2015) for clustering cells, most of these

79 methods are unsupervised and unable to identify cell populations automatically. 
80 For scRNA-seq data, a number of methods have been developed to assign cells to different

81 cell types based on expression profiles. For example, SingleR(Aran et al. 2019) trains on an

82 extensive collection of large annotated reference transcriptomics single cell data.

83 Preliminary labeling by SingleR can vastly accelerate cell type inference. However, there is 84 no similar automated tool for single cell proteomics data annotation. A recently developed

85 tool named CellGrid(Chen et al. 2020) applied the same idea to CyTOF data but needed a

86 large number of labeled proteomics datasets as input. However, there is a lack of labeled

87 single cell proteomics data although well-annotated scRNA-seq data are more broadly

88 available. For the CITE-seq data, it is still necessary to annotate the transcriptomics and

89 proteomics data separately.

90 To overcome the lack of labeled single cell proteomics data, we introduce an automated

91 single cell proteomics data annotation approach called ProtAnno, based on non-negative

92 matrix factorization (NMF) to incorporate data from different reference sources. The only

93 essential input of ProtAnno is some prior knowledge on cell type-specific biomarkers. To

94 further improve annotation accuracy, ProtAnno can take advantage of publicly available

95 CITE-seq data and annotated scRNA-seq data. This enables ProtAnno to perform cell type

96 annotation with no prior characterization between cell types and surface proteins by

97 leveraging these external references.

98 We have evaluated the performance of ProtAnno through simulations under different

99 settings. The results showed the robustness of ProtAnno to biological variability, technical

100 noise, cell type number, and incomplete and inaccurate expert knowledge. We then applied

101 ProtAnno to three real datasets: peripheral blood mononuclear cell (PBMC) paired

102 stimulated B cell receptor CyTOF data, PBMC CITE-seq data from healthy subjects, and

103 longitudinal whole blood covid-19 CyTOF data grouped by patients' disease severity. In the

104 analyses of these real data, ProtAnno provided fast and accurate labeling as demonstrated

105 through comparisons with manual annotations or downstream biological investigations. In

106 summary, ProtAnno is a computationally efficient and statistically robust approach for

107 automated cell type annotation when only limited expert knowledge is available for single

108 cell proteomics data. 


\section{Methods}

\section{Automated Single cell Proteomics Data Annotation Model}

115 ProtAnno deconvolutes the proteomic expression profile $X \in R^{D \times C}$ into the product of the 116 cell type-specific signature matrix, $W \in R^{D \times K}$, and cell type assignment matrix, $H \in R^{K \times C}$, 117 i.e. $X=W H$. In the model, we have $D$ surface markers for $C$ cells in the proteomics data, e.g., 118 the cytometry data and antibody profile in CITE-seq. We denote $K$ as the number of cell 119 types. The columns of $W$ are matched with the known cell types in the same order. Due to 120 the non-negative requirement on the estimations of $W$ and $H$, ProtAnno implemented NMF 121 for solving $X=W H$.

122 In ProtAnno, we integrate information from both prior knowledge encoded in a matrix $A_{0}$ 123 and relationship between protein markers and RNA-seq data encoded in a matrix $A$. In our 124 model, we denote $A \in R^{D \times G}$ as the protein-RNA association matrix inferred from a CITE125 seq data by elastic net [39], where $G$ is the number of genes considered. A desirable CITE126 seq data should include a large number of measured antibodies. ProtAnno implements two 127 internal dictionary-like CITE-seq datasets from Unterman et al. (2020)(Unterman et al. 128 2020) and Ramaswamy et al. (2020) (Ramaswamy et al. 2021). Both datasets have more than 180 antibody tags. The transcriptome signature matrix $S \in R^{G \times K}$ is generated by wellannotated scRNA-seq data. The $K$ columns of $S$ should be matched with $W$. We recommend using the denoised scRNA-seq data to impute drop-out events for better annotation. Specifically, we deploy SAVERx(J. Wang et al. 2019) for imputation due to its superior 133 performance.

134 The expert knowledge matrix $A_{0} \in R^{D \times K}$ is designed based on known cell type surface 135 markers. $A_{0}$ is a discrete matrix containing three possible values, $+1,-1$, and 0 . If 136 biomarker $i$ should have high expression level in cell type $j$, we set $A_{0}^{i j}=1$; if the

137 biomarker is not expressed in this cell type, $A_{0}^{i j}=-1$; and if there is no constraint on the 138 biomarker and cell type, then we set $A_{0}^{i j}=0$.

139 To estimate the signature matrix $W$ in the order of given cell type list, ProtAnno adds 140 constraint on $W$ with respect to the above two proteomic signature matrices, $A S$ and $A_{0}$.

141 With all the notations introduced above, the ProtAnno model is formulated as follows:

$$
\begin{gathered}
\min _{W \geq 0, H \geq 0} L(W, H)=\frac{1}{2}\|X-W H\|^{2}{ }_{F}-\lambda_{1} \operatorname{tr}\left(W^{T} A S\right)-\lambda_{2} \operatorname{tr}\left(W^{T} A_{0}\right)+\frac{\mu}{2}\|W\|_{F}^{2}+\frac{\eta}{2} \\
\left\|1_{k}^{T} H-1_{N}^{T}\right\|^{2}{ }_{2}
\end{gathered}
$$

142 Note that ProtAnno adds regularization penalty on $W$ and $H$ to improve performance. We 143 use $1_{k}$ and $1_{N}$ to denote the $K$-dimensional and $N$-dimensional column vectors with all- 
144 ones. The fourth term in ProtAnno is to control the scale of $W$, and the last term is to force 145 the column sum of $H$ to be 1.

146 We optimize this overall objective function based on the multiplicative update 147 algorithm(Zhang et al. 2008; Wu and Wang 2014) to guarantee non-negativity. The 148 algorithm requires the specifications of the penalty parameters, $\lambda_{1}, \lambda_{2}, \mu$, and $\eta$. In each 149 iteration, ProtAnno updates $\mathrm{W}$ by rows and $\mathrm{H}$ by columns. The details of ProtAnno are 150 provided in Algorithm 1.

151 Model input: Normalized expression profile matrix $X$, discrete prior knowledge matrix $A_{0}$, 152 public labeled single cell expression profile, and penalty parameters $\lambda_{1}, \lambda, \mu, \eta$.

153 Step 1: Set $t=0$ and generate the initial non-negative $W$ and $H$ with all ones.

154 Step 2: Update $W$ by rows and $H$ by columns:

$$
\begin{gathered}
w_{i j}^{t+1}=w_{i j}^{t} \frac{\left[x H^{T}\right]_{j}^{+}+\lambda_{1}\left[(A S)_{i}\right]_{j}^{+}+\lambda_{2}\left[\left(A_{0}\right)_{i}\right]_{j}^{+}}{\left[w_{i}\left(H H^{T}+\mu I\right)\right]_{j}+\left[x H^{T}\right]_{j}^{-}+\lambda_{1}\left[(A S)_{i}\right]_{j}^{-}+\lambda_{2}\left[\left(A_{0}\right)_{i}\right]_{j}^{-}} \\
h_{j}^{t+1}=h_{j}^{t} \frac{\left[W^{T} x\right]_{j}^{+}+\eta 1_{k}}{\left[\left(W^{T} W+\eta 1_{k} 1_{k}^{T}\right) h^{t}\right]_{j}+\left[W^{T} x\right]_{j}^{-}}
\end{gathered}
$$

Step 3: Repeat Step 2 until the convergence or reaching the pre-specified number of

157 iterations.

158 It can be shown that the algorithm converges in theory with the details of the theorems and converging rates provided in the supplementary materials.

\section{Choices of Penalty Parameters}

161 Since the penalty parameter values are critical to ProtAnno, we developed the following

162 algorithm to tune their values iteratively.

163 To ensure that ProtAnno can group expression profiles into reasonable clusters, we use the 164 default unsupervised Louvain algorithm for preliminary clustering. We then set the initial 165 parameter $\eta$ by the KKT condition; and then search the initial $\lambda_{1}, \lambda_{2}$, and $\mu$ values by 166 choosing from among $0.1,1,10$, and 100 . To find the initial $\lambda_{1}$ and $\lambda_{2}$ values, we maximize 167 the ARI between the Louvain clusterings and ProtAnno results. We consider a novel index 168 to select the initial value for $\mu$ :

$$
D(\mu):=X_{\text {mean }}-W_{\text {mean }} .
$$

170 The above function considers the difference between the mean values of $X$ and $W$. The 171 main purpose of $D(\mu)$ is to ensure that $W$ is on approximately the same scale as $X$. 
172 Otherwise, the deconvolution would be unstable since we penalize the column summation

173 of $\mathrm{H}$ to make the cell type proportion estimations meaningful.

174 To set more precise penalty terms, ProtAnno uses binary search to determine the final 175 optimal output as detailed in Algorithm 2, and allows the specified search depth depending 176 on the running time. If the final ARI is lower than 0.75 , the algorithm will restart the binary 177 search at higher resolution.

178 Model input: Normalized expression profile matrix $X$, discrete prior knowledge matrix $A_{0}$, 179 publicly labeled single cell expression profile.

180 Step 1: Estimate Louvain clusters.

181 Step 2: Initialize $W$ and $H$ by an optimization method of choice $\left(\lambda_{1}=1\right.$ and $\left.\lambda_{2}=10\right)$, and 182 initialize $\eta$ by KKT conditions:

$$
\eta:=\left\|\left(W^{T} W H-W^{T} X\right) /\left(1_{K} 1_{K}^{T} H-1_{K} 1_{N}^{T}\right)\right\|_{\text {median }} .
$$

184

185 Step 3: Initialize $\lambda_{1}$ and $\lambda_{2}$ by choosing from $(0.1,1,10,100)$ and minimize Adjusted Rank 186 Index (ARI) with Louvain clustering.

187 Step 4: Initialize $\mu$ by estimated signature matrix W reliability by the following metric:

$$
D(\mu):=X_{\text {mean }}-W_{\text {mean }}
$$

190 Step 5: Select the best $\lambda_{1}, \lambda_{2}$, and $\mu$ by binary search.

191 Step 6 (Optional): If the final ARI with Louvain cluster is still lower than 0.75, we re-do the 192 binary search on all the penalty parameters. 


\section{Results}

\section{Simulation Setup}

200 In the following, we describe how we simulated the protein expression profiles for each

201 single cell. We first generated the entries in the expert matrix $A_{0}$ from a multinomial

202 distribution with three categories, $+1,-1$, and 0 , corresponding to biomarker knowledge for

203 a cell type, i.e., high expression, no expression, and no information. The matrix will be built

204 with the angles between vectors as large as possible, so that the simulated single cell

205 expression profile is a nonnegative linear combination of the proteomics signature

206 matrix(Zhang et al. 2008). To achieve it, we generate 100 matrices randomly and minmax

207 the inner products between column vectors to get the optimal one. This design captures the nature of signature genes. However, the prior knowledge may not be in line with true relationships between markers and cell types due to technical and biological variations. We, therefore, regenerated an intermediate discrete matrix $\tilde{A}_{0}$ based on $A_{0}$ containing three possible discrete values, 2 (high expression), 1 (low expression), and 0 (no expression), by random walk and the protocols observed in real data (details in Methods).

213 We then derived the signature matrix $W$ based on $\tilde{A}_{0}$ from two truncated normal

214 distributions with high and low mean values, respectively, and varying relationships

215 between variances and means that reflect the signal noise ratio for a biomarker. The

216 difference of the means of the two truncated normal distributions together with their

217 variances dictate the informativeness of a specific biomarker for cell type specification. The

218 proteomics expression profile $X$ was sampled from the truncated normal distributions

219 based on the signature matrix that defines the mean and associated variance for each

220 biomarker in each cell type. Finally, to simulate the predicted proteomics signature matrix

221 from transcriptomics data, which is the product of the protein-RNA association matrix $A$,

222 and the transcriptomics signature matrix $S,[A S]$, we sampled the element $[A S]_{i j}$ in the 
223 matrix with mean $W_{i j}$, the expression level of signature gene $i$ in cell type $j$, and variance

$224 W_{i j} /(2 *$ corr $)$. A smaller corr results in a weaker correlation between the two proteomics

225 signature matrices. All the simulation details can be found in Materials and Methods.

\section{Model performances and comparisons}

227 We considered six simulation scenarios to cover a wide range of biological and technical 228 variations in real data, with data quality varying from high (scenario 1) to low (scenario 6).

229 We tuned the parameters of the normal distributions to decrease the cell type distinction 230 and enlarge the expression profile divergence. To simulate the situations when AS or is

231 too noisy to have a good prediction power, we also added more uncertainty to AS and

232 that led to a lower correlation with real signature matrix $\mathrm{W}$. The overall noise increased

233 from scenario 1 to 6 . In scenario 5, we increased the noisy level of expert matrix so that

234 it is more aberrant compared with the single cell proteomics data expression pattern. In

235 addition, the correlation of and was set at only 0.2. These large noises from

236 references would reduce the annotation accuracy. Furthermore, we increased expression

237 profile variations by lowering the mean-variance ratio in scenario 6, making the annotation 238 more challenging. All the parameters settings and details are listed in Supplementary Table

2391.

241 We compared the performance of six models: the full ProtAnno model, the ProtAnno model without transcriptomics information $A S$, the ProtAnno model without expert knowledge

$243 A_{0}$, the unsupervised ProtAnno model, the unsupervised Louvain clustering, and cell type

244 assignment by non-negative least squares (NNLS) when the protein signature matrix $W$ is

245 known. The last one is the best result an NMF model can achieve. We consider five metrics

246 for annotation accuracy: Adjusted Rand Index (ARI), annotation accuracy assigned by the

247 largest value for every single cell, Normalized Mutual Information (NMI), cosine similarity

248 between the estimated and real assignment, and Average Silhouette Width.

249 The simulation results in Figure 2A show that the full ProtAnno model achieved the best

250 annotation accuracy, especially compared with the NNLS model. Specifically, from

251 scenarios 1 to 4 , ProtAnno was able to achieve the same performance an NNLS model could

252 have when the true signature matrix is known. These results suggest the importance of

253 having precise information for $A_{0}$ and $A S$. Besides the full ProtAnno model, the partial

254 ProtAnno model with the expert matrix had the second-best performance, just slightly

255 worse than the full model. The partial ProtAnno model only having the transcriptomics

256 reference was not competitive with the first two models. But it was still much better than

257 the unsupervised clustering, even in terms of ARI and NMI. In general, both full and partial

258 ProtAnno models could obtain accurate cell type assignments even in the presence of

259 considerable noise. 
We investigated the numerical convergence of ProtAnno in our simulation studies. Fig 2B shows an example of ProtAnno cell type assignment in a UMAP plot with 29 cell types, where the accuracy was $98.8 \%$. The high agreement of clustering between the real labels (left) and the ProtAnno annotation (right) demonstrates the power of ProtAnno.

\section{Algorithm convergence and robustness}

ProtAnno generally converges after 100 iterations (Supp 1A). To filter the high confidence annotation, the subsetting step will keep the cell whose estimated $H$ is greater than 0.5 after column normalization. The subsetting step was able to slightly enhance the annotation accuracy (Supp 1B).

ProtAnno's robustness was investigated with respect to three parameters: mean-variance ratio (Fig 3A), correlation between $W$ and AS (Fig 3A, supp 1C), and cell type number $K$ (Fig 3C). Since a protein with a higher mean expression level tends to have a higher variance, we used mean-variance ratio to characterize the signal noise ratio of a protein. As expected, the prediction accuracy dropped with a reduced mean-variance ratio (Fig 3A). Overall, the full ProtAnno model was very robust even when the $A S$ prediction power was weak (supp 1C), implying the critical role of $A_{0}$. We also explored how the transcriptomics information $A S$ could help the annotation when $A_{0}$ is not available (supp 1C). The accuracy rate was almost linearly associated with the prediction correlation. Therefore, scRNA-seq data and dictionary CITE-seq data with high quality are essential for the good performance of the partial ProtAnno model when no reliable expert knowledge is available. As for the impact of the number of cell types, ProtAnno was robust to the large cell type number as long as the cell counts of each cell population were more than 100 in our simulation (Fig 2B, Fig 3C).

The good performance of ProtAnno depends on the appropriate choices of the penalty parameters. Simulation results suggest that our proposed parameter tuning algorithm was able to find good penalty values (Fig 3D, Supp 1D) as shown in the relationship between algorithm performance and tuning parameter values in these figures in most cases.

Finally, we can rewrite the ProtAnno objective function in an equivalent form as (see supplementary materials):

$$
\min _{W \geq 0, H \geq 0} \frac{1}{2}\|X-W H\|_{F}^{2}+\frac{\mu}{4}\left\|W-\frac{2 \lambda_{1}}{\mu}(A S)\right\|_{F}^{2}+\frac{\mu}{4}\left\|W-\frac{2 \lambda_{2}}{\mu} A_{0}\right\|_{F}^{2}+\frac{\eta}{2}\left\|1_{k}^{T} H-1_{N}^{T}\right\|_{F}^{2} .
$$

291 The above objective function form illustrates the imposed relationships between W, AS,

292 and $A_{0}$. The second penalty terms will force the ratio of elements in matrices W and AS to 
293 be approximately equal to $\frac{2 \lambda_{1}}{\mu}$. Specifically, the ratio of $W_{i j} /[A S]_{i j}$ should be approximately

$294 \frac{2 \lambda_{1}}{\mu}$ for any entry with row index $i$ and column index $j$. Similarly, the ratio of W and $A_{0}$

295 should be approximately $\frac{2 \lambda_{2}}{\mu}$. Therefore, we empirically examined whether the ratios of

296 matched elements in matrices W and AS (or $A_{0}$ ) approached the theoretical ones (Supp 2A,

297 2B). The figures show that the empirical distribution of these ratios compared with $\frac{2 \lambda_{1}}{\mu}$ and

$298 \frac{2 \lambda_{2}}{\mu}$, suggesting that estimated $W$ was close to $\frac{2 \lambda_{1}}{\mu}(A S)$ and $\frac{2 \lambda_{2}}{\mu} A_{0}$ at the same time.

\section{cytometry data}

We first evaluated ProtAnno's performance on a benchmarking BCR CyTOF dataset with eight paired samples from PBMC in Bodenmiller et al. (2012)(Bodenmiller et al. 2012). Compared with the reference group, the B cell population was stimulated by BCR in the treatment group. All the samples were collected from healthy individuals, and 10 cell type markers were measured in a total of 172,791 cells. This dataset was labeled in Nowicka et al. (2017)(Nowicka et al. 2017) after the authors applied an unsupervised clustering method FlowSOM(Van Gassen et al. 2015) and manually merged clusters. We evaluated the assignments of these cells to five major cell types based on the 10 cell type markers. Other inputs, the association matrix and the transcriptomics signature matrix $\mathrm{S}$, were generated by the CITE-seq data in Ramaswamy et al. (2020)(Ramaswamy et al. 2021). To match with annotated cell types by Nowicka et al., we manually gated the five major cell types in the scRNA-seq. We also constructed an expert-guided matrix $A_{0}$ (Table 1 ). ProtAnno assigned a cell to the cell type with the largest value in matrix $\mathrm{H}$.

316 The overall median accuracy of ProtAnno for this data set was 83\% (Fig 4A) across the samples. For most of the samples, the accuracy could be as high as $80 \%$ to $85 \%$ (Supp3), except an outlier, patient 8, with decreased the average. For this data set, even the partial ProtAnno with only $A_{0}$ or only $A S$ could achieve an accuracy of over $70 \%$ overall. The subsetting step could slightly improve accuracy by keeping high-confidence cells (Supp 3, Supp 4). Because the BCR dataset does not have clear clustering patterns, making it challenging to annotate cells. This can be seen in the UMAP plot, where some of the cells from different cell types are located in the same region (Fig 4E). Similar observation was made in the benchmarking study of unsupervised clustering accuracy by Weber et al.

325 (2019)(Weber et al. 2019). At the lowest clustering resolution of 9 clusters, the false 326 positive rate was around $20 \%$, where unsupervised clustering could not achieve accurate 
annotation. Therefore, an overall accuracy of $80 \%$ may be considered satisfactory for this dataset. We further investigated the misclassification patterns by the confusion matrix for patient 1 in the stimulated group (Fig 4B). It can be seen that most of the cells could be correctly assigned by ProtAnno. However, some of the natural killer cells (NK cells) were annotated as CD8 T cells. That is partly because the BCR data did not measure the biomarker CD8, which offers important information for CD8 T cell assignment. For this data set, the only biomarker that was informative to distinguish these two cell populations is CD3, which makes computational annotation difficult. The UMAP plot also shows that the annotation is highly consistent with the manually annotated cell types, although the boundary of NK cells and CD8 T cells is hard to recognize (Fig 4E).

Since many genes had significant differential expression levels across groups in the BCR dataset, we studied the $\mathrm{W}$ matrix variations to test whether ProtAnno could accurately estimate the signature matrix. The most differential expression directions in the stimulated group by ProtAnno are consistent with prior biological knowledge (Fig 4C). For example, biomarker HLA-DR had a significantly elevated expression in most cell types after BCR stimulation. The inferred cell type proportions also suggest that ProtAnno can provide an accurate estimation in the comparative study. For example, the proportions of naive B cells were lower in the stimulated group (Fig 4D). These downstream analyses suggest accurate

345 annotation by ProtAnno.

To investigate the performance of ProtAnno on the proteomic expression profile in CITEseq data, we analyzed a PBMC CITE-seq dataset reported in Wang et al. (2020)(X. Wang et al. 2020). This dataset measured 10 surface markers in 1372 cells from a healthy subject. We used ProtAnno to annotate 1153 cells with six cell types, because we cannot annotate the other 219 cells to any cell type based on the available limited 10 markers. These cells were manually labeled and the details are provided in the supplementary materials. We

354 used the CITE-seq data in Unterman et al. (2020)(Unterman et al. 2020) to derive the transcriptomics signature matrix and the CITE-seq data in Ramaswamy et al.

356 (2020)(Ramaswamy et al. 2021) to obtain the association matrix and construct $A_{0}$ (Table

357 2). The automated annotation accuracy was around $99 \%$ with $100 \%$ accuracy for most cell

358 types, except for non-classical monocytes. ProtAnno failed to recognize this cell type since

359 it is a rare population whose cell type proportion is below 1\% (Fig 5A). These results

360 suggest that ProtAnno could be applied to multiple single cell proteomics sequencing

361 platforms, though cytometry and CITE-seq are different technologies with distinct features.

\section{Application to longitudinal whole blood covid-19 cytometry data}


364 Finally, we applied ProtAnno to a longitudinal whole blood covid-19 CyTOF data set reported in Rodriguez et al. (2020)(Rodriguez et al. 2020). This dataset included 37 adult covid-19 patients classified into ICU, non-ICU, and recovery groups. All the hospitalized (ICU and non-ICU) patients were measured at multiple time points. Each sample had 15,000 20,000 cells. The authors used an in-house annotation method CellGrid(Chen et al. 2020 ) to train a subtyping strategy with high resolution to annotate over 50 cell types. Since we do not have access to such a large training CyTOF dataset and comprehensive knowledge on biomarkers, we selected 23 surface markers to define 17 common cell types in whole blood. For this dataset, we only considered the partial ProtAnno model with $A_{0}$ since there is no whole blood CITE-seq dataset available (Table 3). Since we do not have ground truth on cell type annotations, we primarily evaluated the performance of ProtAnno through downstream analysis. More specifically, we studied the cell counts and cell types of neutrophils and lymphocytes.

377 The neutrophiles are strongly positively correlated with patient disease severity

378 (Aschenbrenner et al. 2021). In this dataset, we treated the patient group as the severity 379 indicator. In the ProtAnno results, the neutrophil cell counts of recovery patients were 380 significantly lower than those of the hospitalized patients (Fig 5B, 5C) with the Wilcoxon test p-value smaller than 0.05 for both raw output and subsetting output of ProtAnno,

382 consistent with our expectation. It is also known that the inflammatory symptoms are 383 dramatically elevated in severe cases(C. Huang et al. 2020; Ong et al. 2020; Su et al. 2020; 384 Hadjadj et al. 2020). Longitudinal analysis of this dataset showed a slight decrease of 385 neutrophils proportions over time after admission, except with one patient, COV-34, having 386 a slight increase at the end of the study (Fig 5D, 5E). The changes between day 1 to day 12 387 were significant, especially for the ICU group with a p-value of 0.029 . These results suggest 388 that most ICU patients were recovered after treatment.

389 In contrast, the lymphocyte proportion is expected to decline in severe covid-19 patients.

390 Lymphopenia has been used as a biomarker to define a patient's morbidity(Fathi and

391 Rezaei 2020; Tavakolpour et al. 2020; I. Huang and Pranata 2020). When we applied

392 ProtAnno to infer CD4 T and CD8 T cells, although there was no statistically significant 393 difference across groups, the CD4 T cell count was indeed elevated if the patients recovered

394 (Fig 5F). In the longitudinal curves of cell counts, almost all the patients had increased CD4 395 T cell and CD8 T cell counts after treatment (Fig 5G). This implies that the lymphopenia 396 was alleviated by treatment.

397 The analysis of this data set shows the usefulness of ProtAnno even when the data are 398 highly noisy. 


\section{Discussion}

402

403

404

405

406

407

408

409

410

411

412

413

414

415

416

417

418

419

420

421

422

423

424

425

426

427

428

429

430

431

432

433

434

435

In this paper, we have developed a computationally efficient and statistically robust method, ProtAnno, for automated annotation for single cell proteomic data. Using protein expression profiles, this computationally efficient method annotates cells based on cell type information gathered through (imprecise) prior knowledge, publicly annotated single cell datasets, and CITE-seq data. ProtAnno only requires simple and accessible references, e.g. publicly annotated scRNA-seq. When only limited references are available, ProtAnno can be applied either without prior biological knowledge or public transcriptomics references. ProtAnno can also resolve heterogeneity among cell populations. For instance, ProtAnno was able to detect signature matrix variations across different sample groups in the BCR study. In real data applications, we showed that we can gain biological insights through downstream analysis, such as cell type-specific differential expression analysis and cell type proportion comparisons after annotations by ProtAnno.

Simulation results showed that ProtAnno is robust to the number of cell types and noises in cytometry and CITE-seq antibody expression profiles. However, ProtAnno may be limited to major cell types and unstable when the data are very noisy. Specifically, it is common that the single cell data does not have a clear separated clustering. For example, ProtAnno does not always have a reliable assignment for all samples (supp 3). When a specific cell type is hard to recognize even for manual gating, ProtAnno may misclassify its entire cell subpopulation. The annotation performance also dropped substantially in simulations with large noise in $\mathrm{X}$ and/or more limited information from references $A_{0}$ and $A S$. Therefore, ProtAnno may be suitable for preliminary assignments and may require manual inspection.

Future work can incorporate hierarchical cell type structure to enhance the classification resolution and accuracy. Specifically, ProtAnno could be extended with multiple layers for classification. The cell populations with low resolution, i.e., neutrophils and lymphocytes, could be identified first, and the cell subtypes, i.e., naive CD4 T cell and memory CD8 T cell, can be classified in the next few steps. Additionally, some steps in the parameter tuning algorithm could be replaced by advanced computational clustering methods, i.e., FlowSOM(Van Gassen et al. 2015) and SPADE(Qiu et al. 2011). This may be helpful when the boundaries of cell types are unclear. Lastly, although ProtAnno does not need training data, a more unbiased association matrix inferred from more data could be a helpful addon item to ProtAnno.

In summary, ProtAnno can provide a robust preliminary automated cell type annotation. Its performance could be further improved through adopting more advanced clustering approaches and more reliable references. 
bioRxiv preprint doi: https://doi.org/10.1101/2021.09.13.460162; this version posted September 13, 2021. The copyright holder for this preprint (which was not certified by peer review) is the author/funder. All rights reserved. No reuse allowed without permission.

\section{$454 \quad$ Figures and Legends}

$455 \quad$ Fig1. Model overview. 
456 ProtAnno first generates a protein-RNA association matrix $A$ trained on CITE-seq

457 dictionary by Elastic Net and a transcriptome signature matrix $S$ from scRNA-seq with cell

458 type annotations. The product of $A$ and $S$ is the predicted protein level signature matrix

459 based on mRNA counts. The other input is the discrete matrix $A_{0}$ to represent the general

460 expert biomarker knowledge. The loss function is an NMF optimization program on protein

461 expression matrix to deconvolute into estimated protein signature matrix $W$ and cell type

462 assignment matrix $H$, with penalizations on $A S$ and $A_{0}$ and regularizations on $W$ scale and

463 column sum of $H$. The matrix $H$ could be optionally trimmed to the cells which have high-

464 confidence assignment in the presence of high noise in $X$. The final annotations of ProtAnno

465 are based on the largest value of $H$ column-wise.

Fig2. ProtAnno simulation benchmarking results.

467 A) Comparisons of benchmarking annotation results under six simulation models. The x-

468 axis lists the six models; the y-axis shows the annotation metric values.

B) An example of ProtAnno annotation when the number of cell types $K=29$. The left panel is the UMAP plot on true labels; the right panel is the UMAP plot of ProtAnno

471 annotations.

\section{Fig3. ProtAnno model robustness evaluations.}

473 A) The relationship between ProtAnno annotation accuracy and expression variation. The

$474 \mathrm{x}$-axis represents the expected ratio of mean and variance. A higher value indicates lower

475 expression variation. The y-axis shows the values of annotation metrics. The curves

476 represent the results from 100 simulations for each mean-variance ratio value.

477 B) The effect of transcriptomics data on a full ProtAnno model. The $\mathrm{x}$-axis represents the

478 correlation between transcriptomics predicted signature matrix $A S$ and real protein

479 signature matrix $W$. The y-axis represents the value of annotation metrics.

480 C) ProtAnno robustness as a function of cell type number $K$. The $x$-axis represents cell type 481 number that varies from 3 to 40 . The $y$-axis represents the value of annotation metrics.

482 D) Evaluation of parameter tuning algorithm. The $\mathrm{x}$-axis represents the change of $\lambda_{1}$ from 0 483 to 5 under six simulation scenarios. The color represents different annotation metrics. The $484 \mathrm{y}$-axis represents the value of annotation metrics. The red vertical line represents the 485 optimal $\lambda_{1}$ by the parameter tuning algorithm for each simulation scenario.

\section{Fig4. ProtAnno results on BCR cytometry data.}

487 A) Benchmarking ProtAnno on five labeled cell types. The x-axis lists the four ProtAnno 488 models; the y-axis is the annotation metric value. 
B) Confusion matrix of ProtAnno annotation on BCR stimulated patient 1. The y-axis is the ProtAnno results compared with the true cell type labels as a reference in the $\mathrm{x}$-axis.

491 C) The boxplots of estimated signature expression in $W$. The $\mathrm{x}$-axis represents the cell type 492 and the $y$-axis is the distribution of values in the signature matrix across patients within

493 groups. The first two columns are ProtAnno annotation results in the stimulated and 494 unstimulated BCR groups and the last two columns are the true average signature 495 expression distributions.

496 D) Cell type proportions boxplots. In this figure, we compare cell type proportions 497 estimated by ProtAnno with true proportions. The first two columns are ProtAnno 498 annotation results in the stimulated and unstimulated BCR groups and the last two 499 columns are the true cell type proportions in these two groups.

500 E) The UMAP plot. The upper panel is the true annotation; the lower panel is the 501 assignment by ProtAnno.

502 Fig5. ProtAnno results on PBMC CITE-seq data and temporal whole blood covid-19 cytometry 503 data.

504 A) The UMAP plot of the proteomic data in the PBMC CITE-seq study in Wang et al. 505 (2020)(X. Wang et al. 2020). The left panel is the true annotation; the right panel is the 506 assignment by ProtAnno. The UMAP projections are colored by the cell types.

507 B) and C) Boxplots of cell type counts of neutrophil cells from the whole blood covid-19 508 study in Rodriguez et al. (2020)(Rodriguez et al. 2020) by ProtAnno across different 509 patient groups i.e., ICU, non-ICU, and recovery patients. B) is the raw output of ProtAnno. C) 510 is the subsetted output of ProtAnno for keeping high-confidence assignments only. The 511 paired Wilcoxon test $p$-values are shown on the top of the figures.

512 D) Neutrophil cell counts estimation by ProtAnno over the days after admission. The color 513 represents the patient who has the longitudinal cytometry data.

514 E) Neutrophil cell type proportions estimated by ProtAnno over the days after admission. 515 Two curves represent the ICU and non-ICU patient groups.

516 F) Boxplots of CD4 T cell and CD8 T cell counts across groups (ICU, non-ICU, and recovery 517 patients).

518 G) CD4 T and CD8 T cell type proportions estimation by ProtAnno over the days after 519 admission. The color represents the patient who has the longitudinal cytometry data.

520 Supp1. ProtAnno optimization properties. 
521 A) ProtAnno convergence for six simulation scenarios. The $x$-axis represents iteration

522 number. The y-axis represents log loss function values.

523 B) The subsetting cutoff parameter. The x-axis represents the cutoff value. The y-axis

524 represents annotation metrics value.

525 C) The impact of transcriptomics data on annotation in the ProtAnno model with only

526 transcriptomics penalization, $A S$. The x-axis represents the correlation between

527 transcriptomics predicted signature matrix $A S$ and true protein signature matrix $W$. The y-

528 axis represents the annotation metrics value.

529 D) The evaluation of parameter tuning algorithm. The $\mathrm{x}$-axis corresponds to $\lambda_{2}$ from 0 to 60

530 under six simulation scenarios. The color represents different annotation metrics. The y-

531 axis represents the annotation metrics value. The red vertical line represents the optimal

$532 \lambda_{2}$ by the parameter tuning algorithm for each simulation scenario.

\section{Supp2. Penalization powers in optimization.}

534 A) The distribution of ratios between estimated $W$ and the input, $A S$. The red vertical line is 535 the median of the distribution. The orange vertical line is the true ratio in the rewritten 536 optimization algorithm.

537 B) The distribution of ratios between estimated $W$ and the input, $A_{0}$. The red vertical line is 538 the median of the distribution. The orange vertical line is the true ratio in the rewritten 539 optimization algorithm.

540 Supp3. The prediction power on BCR data.

541 The annotation accuracy barplots across samples from stimulated (first row) and 542 unstimulated (second row) groups. The columns are the cell type numbers used ( 5 cell 543 types in the first column, 6 cell types in the second column, and 7 cell types in the third 544 column). The color represents the raw output or subsetted output from ProtAnno.

545 Supp4. Annotation accuracy improvement by subsetting.

546 A) The annotation metrics with different subsetting cutoffs. The $\mathrm{x}$-axis represents the cutoff 547 value. The y-axis represents the annotation metric value.

548 B) The numbers of kept cells after subsetting filter. The x-axis represents the cutoff value. 549 The y-axis represents the remaining cell counts.

550 Supp5. The violin plots of estimated signature expression in in a longitudinal covid-19 study 551 by ProtAnno. 
552 The $\mathrm{x}$-axis represents the cell type and the $y$-axis is the distribution of values in the

553 signature matrix across patients within groups. The colors represent patient groups (ICU

554 and recovery).

555

556

557

558

559

560

561

562

563 Materials and Methods

\section{Optimization Procedure}

\section{Step 1: Update $w$ with Lagrangian Multiplier}

566 In this step, we update the k-dimensional non-negative row vector $w_{i}$. Thus we have the

567 new loss function by adding the Lagrangian multiplier as equation

568 [eq:updateWlossfunction]. Here to discriminate the primary constraint optimization with 569 the dual Lagrangian function, we use $E$ to denote the latter.

$$
\min _{w_{i} \geq 0} L\left(w_{i} ; H\right)=\frac{1}{2}\left\|x_{i}-w_{i} H\right\|_{F}^{2}-\lambda_{1} \operatorname{tr}\left(w_{i}^{T}(A S)_{i}\right)-\lambda_{2} \operatorname{tr}\left(w_{i}^{T}\left(A_{0}\right)_{i}\right)+\frac{\mu}{2}\left\|w_{i}\right\|^{2}
$$




$$
\min _{w_{i}} E\left(w_{i} ; H\right)=\frac{1}{2}\left\|x_{i}-w_{i} H\right\|_{F}^{2}-\lambda_{1} \operatorname{tr}\left(w_{i}^{T}(A S)_{i}\right)-\lambda_{2} \operatorname{tr}\left(w_{i}^{T}\left(A_{0}\right)_{i}\right)+\frac{\mu}{2}\left\|w_{i}\right\|^{2}+\Phi w_{i}
$$

570 Here $x_{i}$ and $w_{i}$ are the $i$ th row of $X$ and $W$, respectively. To minimize the loss function by

571 updating row $w_{i}$, we have

$$
\nabla E\left(w_{i} ; H\right)=w_{i}\left(H H^{T}+\mu I\right)-x_{i} H^{T}-\lambda_{1}(A S)_{i}-\lambda_{2}\left(A_{0}\right)_{i}+\Phi .
$$

572 By using the property of $\Phi w_{i}=0$, we have

$$
\begin{gathered}
w_{i j} \nabla E\left(w_{i} ; H\right)_{j}=w_{i j}\left[w_{i}\left(H H^{T}+\mu I\right)-x_{i} H^{T}-\lambda_{1}(A S)_{i}-\lambda_{2}\left(A_{0}\right)_{i}+\Phi\right. \\
\left.=w_{i j}\left[w_{i}\left(H H^{T}+\mu I\right)-x_{i} H^{T}-\lambda_{1}(A S)_{i}\right]_{j}-\lambda_{2}\left(A_{0}\right)_{i}\right]_{j} .
\end{gathered}
$$

574 We set the derivative to be zero. To get the non-negative minimizer, we decompose the

575 items that are not non-negative in [eq:2] into positive and negative parts as:

$$
\begin{gathered}
x_{i} H^{T}=\left[x_{i} H^{T}\right]^{+}-\left[x_{i} H^{T}\right]^{-} \lambda_{1}(A S)_{i}=\left[\lambda_{1}(A S)_{i}\right]^{+}-\left[\lambda_{1}(A S)_{i}\right]^{-} \lambda_{2}\left(A_{0}\right)_{i} \\
=\left[\lambda_{2}\left(A_{0}\right)_{i}\right]^{+}-\left[\lambda_{2}\left(A_{0}\right)_{i}\right]^{-}
\end{gathered}
$$

576 By simple algebra, we have the following equation

$$
\begin{gathered}
w_{i j}\left(\left[w_{i}\left(H H^{T}+\mu I\right)\right]+\left[x_{i} H^{T}\right]^{-}+\left[\lambda_{1}(A S)_{i}\right]^{-}+\left[\lambda_{2}\left(A_{0}\right)_{i}\right]^{-}\right)_{j}=w_{i j}\left(\left[x_{i} H^{T}\right]^{+}+\left[\lambda_{1}(A S)_{i}\right]^{+}+\right. \\
\left.\left[\lambda_{2}\left(A_{0}\right)_{i}\right]^{+}\right)_{j} .
\end{gathered}
$$

579 Thus, we update $w_{i}$ by

580

$$
w_{i j}^{t+1}=w_{i j}^{t} \frac{\left[x H^{T}\right]_{j}^{+}+\lambda_{1}\left[(A S)_{i}\right]_{j}^{+}+\lambda_{2}\left[\left(A_{0}\right)_{i}\right]_{j}^{+}}{\left[w_{i}\left(H H^{T}+\mu I\right)\right]_{j}+\left[x H^{T}\right]_{j}^{-}+\lambda_{1}\left[(A S)_{i}\right]_{j}^{-}+\lambda_{2}\left[\left(A_{0}\right)_{i}\right]_{j}^{-}+10^{-16}} .
$$

\section{Step 2: Update $H$ with multiplicative update algorithm}

582 In this step, we optimize $h$ column-wise.

$$
\min _{h_{j} \geq 0} L\left(h_{j} ; W\right)=\frac{1}{2}\left\|x_{j}-W h_{j}\right\|_{F}^{2}+\frac{\eta}{2}\left\|1_{k}^{T} h_{j}-1_{N}^{T}\right\|_{2}^{2},
$$

584 where $W$ is the constant matrix and $x_{j}$ is the $j$-th column vector of $X$. Similarly, by adding 585 the Lagrangian multiplier, we have the following equations.

$$
\begin{gathered}
\min _{h_{j}} E\left(h_{j} ; W\right)=\frac{1}{2}\left\|x_{j}-W h_{j}\right\|_{F}^{2}+\frac{\eta}{2}\left\|1_{k}^{T} h_{j}-1_{N}^{T}\right\|^{2}{ }_{2}+\Phi h_{j} \\
\nabla E\left(h_{j} ; W\right)=\left(W^{T} W+\eta 1_{k} 1_{k}^{T}\right) h_{j}-W^{T} x_{j}-\eta 1_{k}+\Phi h_{i j} \nabla E\left(h_{j} ; W\right)=0
\end{gathered}
$$

586 We can update by 


$$
h_{j}^{t+1}=h_{j}^{t} \frac{\left[W^{T} x\right]_{j}^{+}+\eta 1_{k}}{\left[\left(W^{T} W+\eta 1_{k} 1_{k}^{T}\right) h^{t}\right]_{j}+\left[W^{T} x\right]_{j}^{-}+10^{-16}} .
$$

\section{Simulation Details}

590 The expert matrix $A_{0}$ was generated from a multinomial distribution containing three 591 categories, $+1,-1$, and 0 . We generated 100 matrices $A_{0}$ and selected the optimal one that 592 had the smallest inner product between the vectors by minimax. Such construction can 593 more likely enable the expression profile $\mathrm{X}$ to be a nonnegative linear combination of basis 594 vectors.

595 In most cases, the expert matrix may be biased. We therefore generated an intermediate 596 discrete matrix $\tilde{A}_{0}$ with three possible discrete values, 2, 1, and 0, to represent the expert 597 knowledge matrix used. They were matched with three biomarker distributions within a 598 cell population: high, low, and no expression. In practice, if the entry in $A_{0}$ was 1 , the 599 corresponding gene would have high expression levels in real data from our observations.

600 Thus, the corresponding element in $\tilde{A}_{0}$ kept the value, 1 . However, if the entry in $A_{0}$ was -1 , 601 it is possible to have low or medium expression level instead of no expression completely. 602 In some rare instances, the corresponding gene might even have high expression.

603 Therefore, the values of -1 would random walk to 1 with probability $q_{-1}$ and to 2 with 604 probability $p_{-1}$ in $\tilde{A}_{0}$. If the entry in $A_{0}$ was 0 , then the distribution was uncertain. In this 605 case, the values of 0 would random walk to 1 with probability $q_{0}$ and to 2 with probability $606 p_{0}$ in $\tilde{A}_{0}$. This generated the new discrete matrix $\tilde{A}_{0}$ based on the above random walk 607 protocols to distinguish the different biomarkers behaviors.

608 Next, the signature matrix $W$ was generated based on $\tilde{A}_{0}$ from two positive truncated 609 normal distributions with different expectations. When the entry in $\tilde{A}_{0}$ was 2 , the 610 corresponding signature gene average expression was sampled from a positive truncated 611 normal distribution with a large mean; when the entry in $\tilde{A}_{0}$ was 1 , the corresponding 612 signature gene average expression was sampled from a half-normal distribution with a 613 small mean; otherwise, it was set to 0.1 .

614 After randomly simulating the cell type labels for each single cell in the proteomics 615 expression profile $X$, the expression level was sampled from normal distributions with the 616 expectation of average signature gene expression in $W$ and variance calculated from 617 sampled mean and the specified mean-variance ratio.

\section{Annotation metrics}


619 ARI was calculated using the function adjustedRandIndex from the R package mclust(Scrucca et al. 2016). For NMI, the ground truth and the predicted label for each cell were converted into one-hot vectors and the function NMI in the R package aricode(Vinh,

622 Epps, and Bailey 2009) was used to calculate the NMI for each cell. The reported result was 623 the average across all the cells. Cosine similarity was calculated by first computing the 624 cosine measure between the ground truth one-hot vector and the cluster assignment vector 625 using the cosine function from the lsa package in $\mathrm{R}$, and finally averaging over all the cells. 626 ASW was calculated using the batch_sil function in the R package kBET(Büttner et al.

627 2018).

\section{Real Datasets and processing}

630 The BCR CyTOF dataset was downloaded through the HDCytoData(Weber and Soneson 2019) R package. The cell types were labeled by Nowicka et al. (2017)(Nowicka et al. 2017) and could be accessed by the HDCytoData package. The CITE-seq data in Wang et al. (2020)(X. Wang et al. 2020) were downloaded from GEO with accession ID GSE148665.

634 The longitudinal covid-19 CyTOF data were downloaded from

635 (https:/ / brodinlab.com/ data-repository/). The two dictionary CITE-seq datasets in Unterman et al. (2020)(Unterman et al. 2020) and Ramaswamy et al. (2020)(Ramaswamy et al. 2021) would be available after they publish the data. The CITE-seq data in Wang et al. (2020)(X. Wang et al. 2020) and Ramaswamy et al. (2020)(Ramaswamy et al. 2021) were manually labeled after the preliminary results by SingleR(Aran et al. 2019). The expertguided matrices were obtained from the biomarker panels in Unterman et al. (2020)(Unterman et al. 2020).

642 All the proteomics data were normalized by arcsinh function with cofactor 5 . We cleaned 643 and gated the longitudinal CyTOF data by the R package flowCore to filter the intact cells. 644 When processing the association matrix from CITE-seq and transcriptomics signature 645 matrix, all the scRNA-seq data are denoised by SAVERX(J. Wang et al. 2019). The 646 transcriptomics gene list for each application contains the matched protein-coding 647 transcript genes (Table 4) and the top highly variable genes generated by the Seurat(Hao et 648 al. 2020) package. al. 2019. "Reference-Based Analysis of Lung Single-Cell Sequencing Reveals a Transitional Profibrotic Macrophage." Nature Immunology 20 (2): 163-72. Antonakos, Melanie Nuesch-Germano, Konstantina Gkizeli, et al. 2021. “Disease Severity- 

Medicine 13 (1): 7.

Bandura, Dmitry R., Vladimir I. Baranov, Olga I. Ornatsky, Alexei Antonov, Robert Kinach, Xudong Lou, Serguei Pavlov, Sergey Vorobiev, John E. Dick, and Scott D. Tanner. 2009. "Mass Cytometry: Technique for Real Time Single Cell Multitarget Immunoassay Based on Inductively Coupled Plasma Time-of-Flight Mass Spectrometry." Analytical Chemistry 81 (16): 6813-22.

Bodenmiller, Bernd, Eli R. Zunder, Rachel Finck, Tiffany J. Chen, Erica S. Savig, Robert V. Bruggner, Erin F. Simonds, et al. 2012. "Multiplexed Mass Cytometry Profiling of Cellular States Perturbed by Small-Molecule Regulators." Nature Biotechnology 30 (9): 858-67.

Büttner, Maren, Zhichao Miao, F. Alexander Wolf, Sarah A. Teichmann, and Fabian J. Theis. 2018. "A Test Metric for Assessing Single-Cell RNA-Seq Batch Correction." Nature Methods 16 (1): 4349.

Chen, Yang, Tadepally Lakshmikanth, Jaromir Mikes, and Petter Brodin. 2020. "Single-Cell Classification Using Learned Cell Phenotypes." bioRxiv, 2020.07.22.216002.

Dong, Xiao, Lei Zhang, Brandon Milholland, Moonsook Lee, Alexander Y. Maslov, Tao Wang, and Jan Vijg. 2017. "Accurate Identification of Single-Nucleotide Variants in Whole-Genome-Amplified Single Cells." Nature Methods 14 (5): 491-93.

Eberwine, James, Jai-Yoon Sul, Tamas Bartfai, and Junhyong Kim. 2014. "The Promise of Single-Cell Sequencing." Nature Methods 11 (1): 25-27.

Fang, Rongxin, Sebastian Preissl, Yang Li, Xiaomeng Hou, Jacinta Lucero, Xinxin Wang, Amir Motamedi, et al. 2021. "Comprehensive Analysis of Single Cell ATAC-Seq Data with SnapATAC." Nature Communications 12 (1): 1337.

Fathi, Nazanin, and Nima Rezaei. 2020. "Lymphopenia in COVID-19: Therapeutic Opportunities." Cell Biology International 44 (9): 1792-97.

Hadjadj, Jérôme, Nader Yatim, Laura Barnabei, Aurélien Corneau, Jeremy Boussier, Nikaïa Smith, Hélène Péré, et al. 2020. "Impaired Type I Interferon Activity and Inflammatory Responses in Severe COVID-19 Patients." Science 369 (6504): 718-24.

Hao, Yuhan, Stephanie Hao, Erica Andersen-Nissen, William M. Mauck, Shiwei Zheng, Andrew Butler, Maddie J. Lee, et al. 2020. "Integrated Analysis of Multimodal Single-Cell Data." bioRxiv, 2020.10.12.335331.

Huang, Chaolin, Yeming Wang, Xingwang Li, Lili Ren, Jianping Zhao, Yi Hu, Li Zhang, et al. 2020. "Clinical Features of Patients Infected with 2019 Novel Coronavirus in Wuhan, China." The Lancet 395 (10223): 497-506.

Huang, Ian, and Raymond Pranata. 2020. "Lymphopenia in Severe Coronavirus Disease-2019 (COVID-19): Systematic Review and Meta-Analysis." Journal of Intensive Care Medicine 8 (May): 36.

Hwang, Byungjin, Ji Hyun Lee, and Duhee Bang. 2018. "Single-Cell RNA Sequencing Technologies and Bioinformatics Pipelines." Experimental \& Molecular Medicine 50 (8): 1-14.

Labib, Mahmoud, and Shana O. Kelley. 2020. "Single-Cell Analysis Targeting the Proteome." Nature Reviews Chemistry 4 (3): 143-58.

Levine, Jacob H., Erin F. Simonds, Sean C. Bendall, Kara L. Davis, El-Ad D. Amir, Michelle D. Tadmor, Oren Litvin, et al. 2015. "Data-Driven Phenotypic Dissection of AML Reveals Progenitor-like Cells That Correlate with Prognosis." Cell 162 (1): 184-97.

Li, Huamin, Uri Shaham, Kelly P. Stanton, Yi Yao, Ruth R. Montgomery, and Yuval Kluger. 2017. "Gating Mass Cytometry Data by Deep Learning." Bioinformatics 33 (21): 3423-30.

Lindeboom, Rik G. H., Aviv Regev, and Sarah A. Teichmann. 2021. "Towards a Human Cell Atlas: Taking Notes from the Past." Trends in Genetics: TIG, April. https://doi.org/10.1016/j.tig.2021.03.007.

Nagano, Takashi, Yaniv Lubling, Tim J. Stevens, Stefan Schoenfelder, Eitan Yaffe, Wendy Dean, Ernest D. Laue, Amos Tanay, and Peter Fraser. 2013. "Single-Cell Hi-C Reveals Cell-to-Cell 
Variability in Chromosome Structure." Nature 502 (7469): 59-64.

Nowicka, Malgorzata, Carsten Krieg, Helena L. Crowell, Lukas M. Weber, Felix J. Hartmann, Silvia Guglietta, Burkhard Becher, Mitchell P. Levesque, and Mark D. Robinson. 2017. "CyTOF Workflow: Differential Discovery in High-Throughput High-Dimensional Cytometry Datasets." F1000Research 6 (May): 748.

Ong, Eugenia Ziying, Yvonne Fu Zi Chan, Wan Ying Leong, Natalie Mei Ying Lee, Shirin Kalimuddin, Salahudeen Mohamed Haja Mohideen, Kian Sing Chan, et al. 2020. "A Dynamic Immune Response Shapes COVID-19 Progression." Cell Host \& Microbe 27 (6): 879-82.e2.

Ornatsky, Olga, Dmitry Bandura, Vladimir Baranov, Mark Nitz, Mitchell A. Winnik, and Scott Tanner. 2010. "Highly Multiparametric Analysis by Mass Cytometry." Journal of Immunological Methods 361 (1-2): 1-20.

Qiu, Peng, Erin F. Simonds, Sean C. Bendall, Kenneth D. Gibbs Jr, Robert V. Bruggner, Michael D. Linderman, Karen Sachs, Garry P. Nolan, and Sylvia K. Plevritis. 2011. "Extracting a Cellular Hierarchy from High-Dimensional Cytometry Data with SPADE." Nature Biotechnology 29 (10): 886-91.

Ramaswamy, Anjali, Nina N. Brodsky, Tomokazu S. Sumida, Michela Comi, Hiromitsu Asashima, Kenneth B. Hoehn, Ningshan Li, et al. 2021. "Immune Dysregulation and Autoreactivity Correlate with Disease Severity in SARS-CoV-2-Associated Multisystem Inflammatory Syndrome in Children." Immunity 54 (5): 1083-95.e7.

Regev, Aviv, Sarah A. Teichmann, Eric S. Lander, Ido Amit, Christophe Benoist, Ewan Birney, Bernd Bodenmiller, et al. 2017. "The Human Cell Atlas." eLife 6 (December). https://doi.org/10.7554/eLife.27041.

Rodriguez, Lucie, Pirkka T. Pekkarinen, Tadepally Lakshmikanth, Ziyang Tan, Camila Rosat Consiglio, Christian Pou, Yang Chen, et al. 2020. "Systems-Level Immunomonitoring from Acute to Recovery Phase of Severe COVID-19." Cell Reports. Medicine 1 (5): 100078.

Scrucca, Luca, Michael Fop, T. Brendan Murphy, and Adrian E. Raftery. 2016. "Mclust 5: Clustering, Classification and Density Estimation Using Gaussian Finite Mixture Models." The R Journal 8 (1): 289-317.

Spitzer, Matthew H., and Garry P. Nolan. 2016. “Mass Cytometry: Single Cells, Many Features." Cell 165 (4): 780-91.

Stoeckius, Marlon, Christoph Hafemeister, William Stephenson, Brian Houck-Loomis, Pratip K. Chattopadhyay, Harold Swerdlow, Rahul Satija, and Peter Smibert. 2017. "Simultaneous Epitope and Transcriptome Measurement in Single Cells." Nature Methods 14 (9): 865-68.

$\mathrm{Su}$, Yapeng, Daniel Chen, Dan Yuan, Christopher Lausted, Jongchan Choi, Chengzhen L. Dai, Valentin Voillet, et al. 2020. "Multi-Omics Resolves a Sharp Disease-State Shift between Mild and Moderate COVID-19." Cell 183 (6): 1479-95.e20.

Tavakolpour, Soheil, Taha Rakhshandehroo, Erin X. Wei, and Mohammad Rashidian. 2020. "Lymphopenia during the COVID-19 Infection: What It Shows and What Can Be Learned." Immunol Lett. https://doi.org/10.1016/j.imlet.2020.06.013.

Unterman, Avraham, Tomokazu S. Sumida, Nima Nouri, Xiting Yan, Amy Y. Zhao, Victor Gasque, Jonas C. Schupp, et al. 2020. "Single-Cell Omics Reveals Dyssynchrony of the Innate and Adaptive Immune System in Progressive COVID-19." medRxiv, 2020.07.16.20153437.

Van Gassen, Sofie, Britt Callebaut, Mary J. Van Helden, Bart N. Lambrecht, Piet Demeester, Tom Dhaene, and Yvan Saeys. 2015. "FlowSOM: Using Self-Organizing Maps for Visualization and Interpretation of Cytometry Data." Cytometry. Part A: The Journal of the International Society for Analytical Cytology 87 (7): 636-45.

Vinh, Nguyen Xuan, Julien Epps, and James Bailey. 2009. Information Theoretic Measures for Clusterings Comparison: Is a Correction for Chance Necessary? Proceedings of the 26th Annual International Conference on Machine Learning.

Wang, Jingshu, Divyansh Agarwal, Mo Huang, Gang Hu, Zilu Zhou, Chengzhong Ye, and Nancy R. 

Methods 16 (9): 875-78.

Wang, Xinjun, Zhe Sun, Yanfu Zhang, Zhongli Xu, Hongyi Xin, Heng Huang, Richard H. Duerr, Kong Chen, Ying Ding, and Wei Chen. 2020. "BREM-SC: A Bayesian Random Effects Mixture Model for Joint Clustering Single Cell Multi-Omics Data." Nucleic Acids Research 48 (11): 5814-24.

Weber, Lukas M., Malgorzata Nowicka, Charlotte Soneson, and Mark D. Robinson. 2019. "Diffcyt: Differential Discovery in High-Dimensional Cytometry via High-Resolution Clustering." Communications Biology 2 (May): 183.

Weber, Lukas M., and Charlotte Soneson. 2019. "HDCytoData: Collection of High-Dimensional Cytometry Benchmark Datasets in Bioconductor Object Formats." F1000Research 8 (August): 1459.

Wu, Siyuan, and Jim Wang. 2014. Nonnegative Matrix Factorization: When Data Is Not Nonnegative. 2014 7th International Conference on Biomedical Engineering and Informatics. IEEE.

Zhang, Junying, Le Wei, Xuerong Feng, Zhen Ma, and Yue Wang. 2008. "Pattern Expression Nonnegative Matrix Factorization: Algorithm and Applications to Blind Source Separation." Computational Intelligence and Neuroscience 2008: 168769.

Zhou, Zilu, Chengzhong Ye, Jingshu Wang, and Nancy R. Zhang. 2020. "Surface Protein Imputation from Single Cell Transcriptomes by Deep Neural Networks." Nature Communications 11 (1): 651. 
Dictionary CITE-seq

RNA

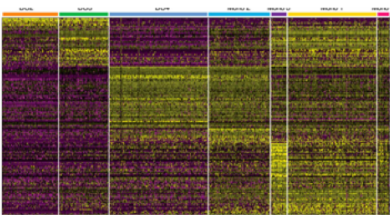

ADT

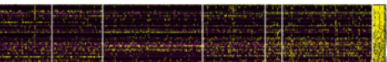

Paired RNA and ADT expression

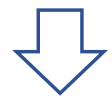

Public

scRNA-seq $S$

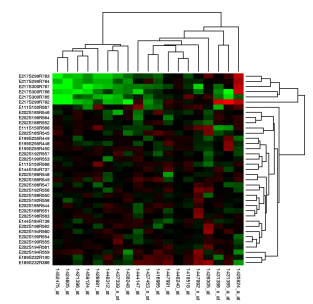

Prior Knowledge on cell type-specific

surface marker

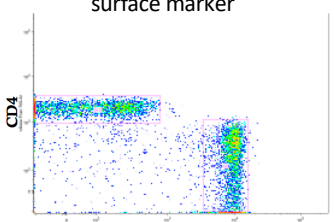

CD8

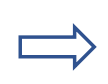

Protein-RNA association $\boldsymbol{A}$

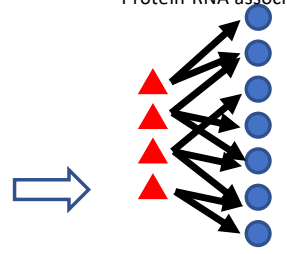

Protein

RNA
Protein

Expression

Profile $\boldsymbol{X}$
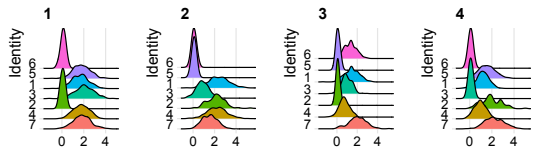

Expression Level Expression Level Expression Level Expression Level
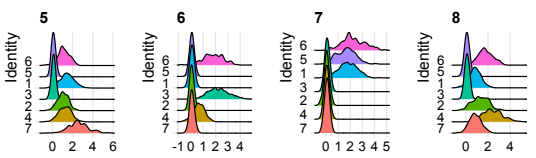

Expression Level Expression Level Expression Level Expression Level

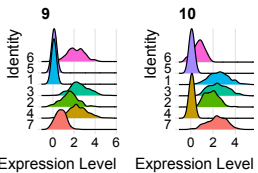

Expression Level Expression Level

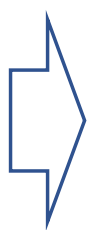

Estimated

Signature Matrix

w

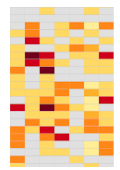

\begin{tabular}{|c|c|c|c|c|}
\hline & \begin{tabular}{|l} 
CD4+ \\
$\mathrm{TT}$
\end{tabular} & \begin{tabular}{|l} 
naîve \\
$B$
\end{tabular} & \begin{tabular}{|l} 
naiive \\
B
\end{tabular} & NK \\
\hline CD4 & 1 & -1 & 10 & 10 \\
\hline CD56 & to & 0 & to & 1 \\
\hline \begin{tabular}{|l|} 
CD3 \\
\end{tabular} & 1 & -1 & -1 & -1 \\
\hline \begin{tabular}{|l|} 
CD19 \\
\end{tabular} & 10 & To & 7 & -1 \\
\hline CD14 & 10 & 10 & 10 & -1 \\
\hline CD11c & & 0 & to & $t_{1}$ \\
\hline \begin{tabular}{|l|} 
CD8 \\
\end{tabular} & -1 & 1 & To & 10 \\
\hline CD16 & 0 & 10 & to & 1 \\
\hline $\mid$ CD127 & & 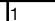 & $T_{0}$ & To \\
\hline
\end{tabular}

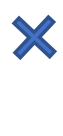

Cell Type

Annotation

Cell Type Assignment $\boldsymbol{H}$ 
A
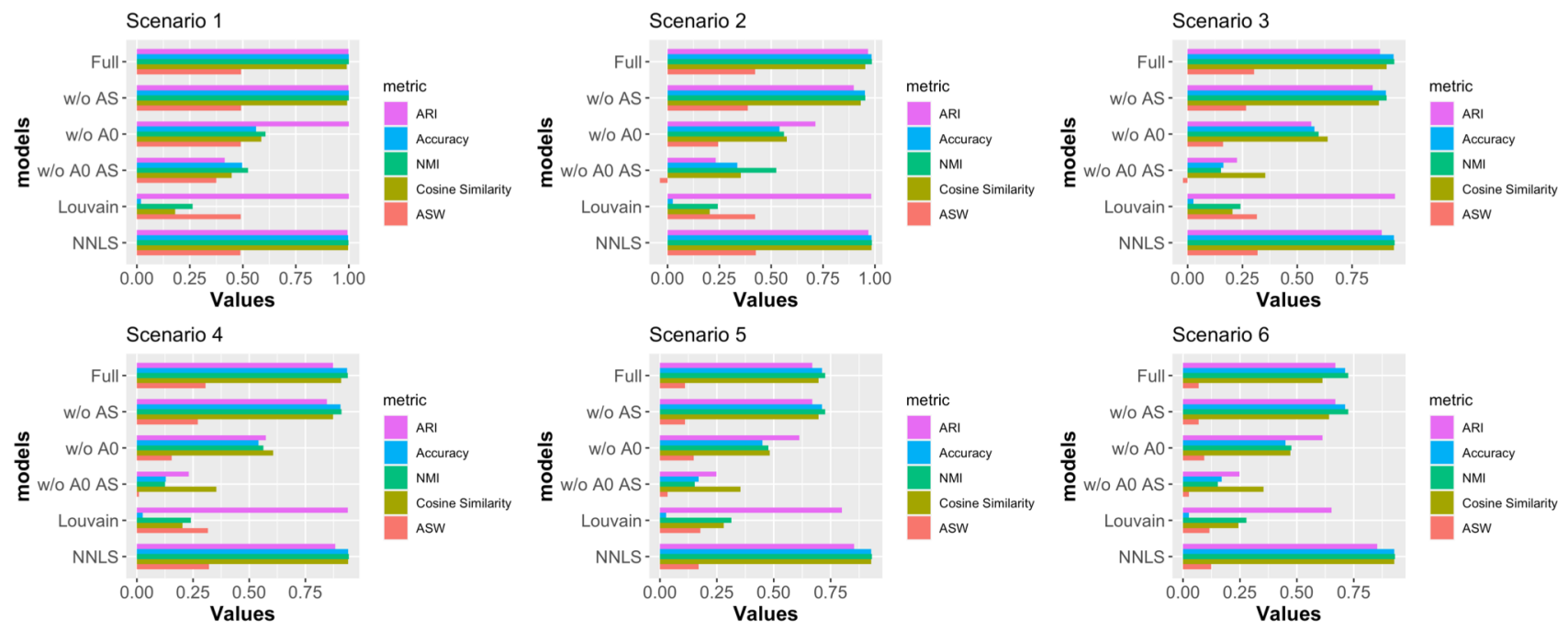

B

label
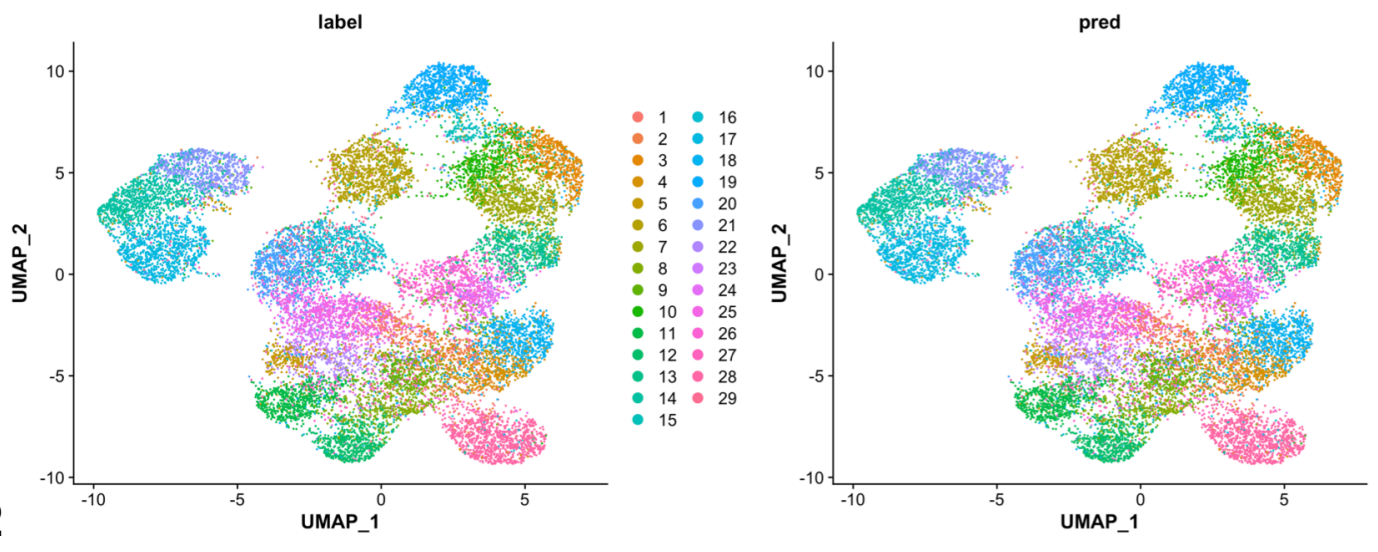

- 1 - 16

$2 \quad 17$

- 3 - 18

- 4 - 19

- 5 - 20

- 6 - 21

- 7 - 22

- 8 - 23

- 9 - 24

- 10 - 25

- 11 - 26

- $12 \cdot 27$

- $13 \cdot 28$

- 14 - 29

15

\section{Fig2}


A

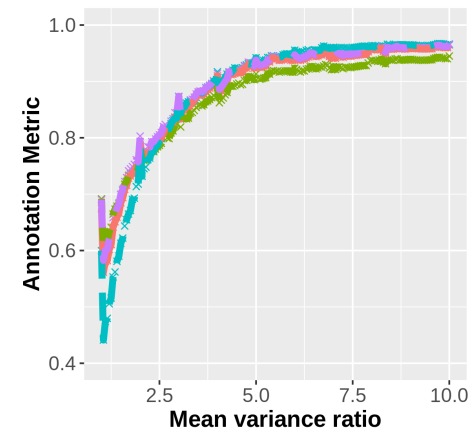

D
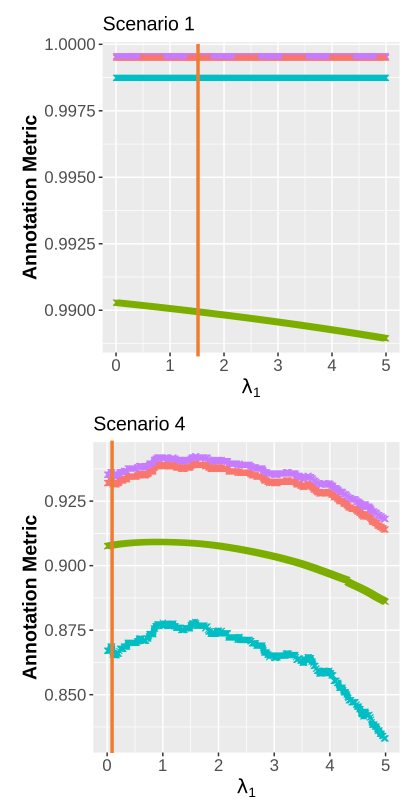

B
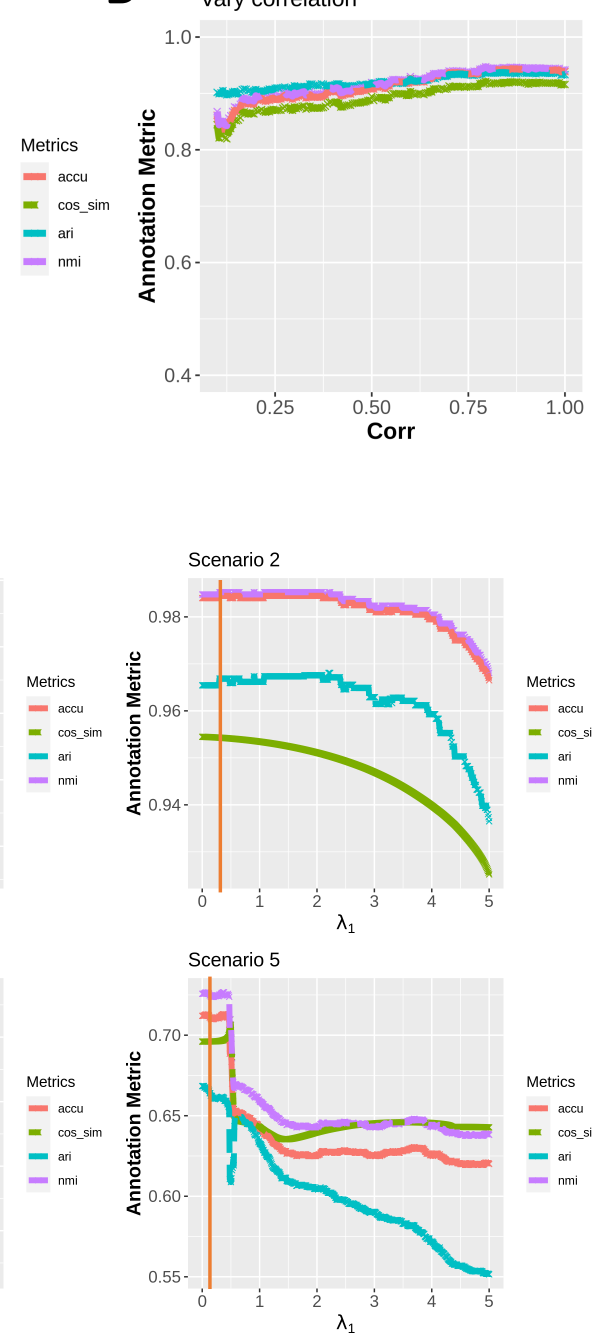

Metrics

$$
\text { - accu }
$$$$
\text { - ari }
$$

C

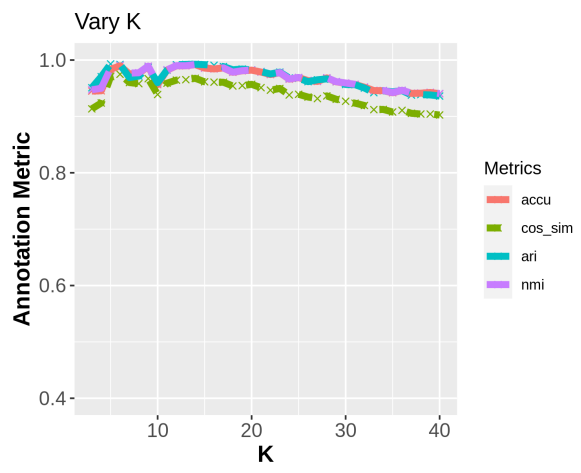$$
\text { = cos_sim }
$$

Metrics

- асси

= cos_sim

- ari

- ${ }^{\mathrm{nmi}}$
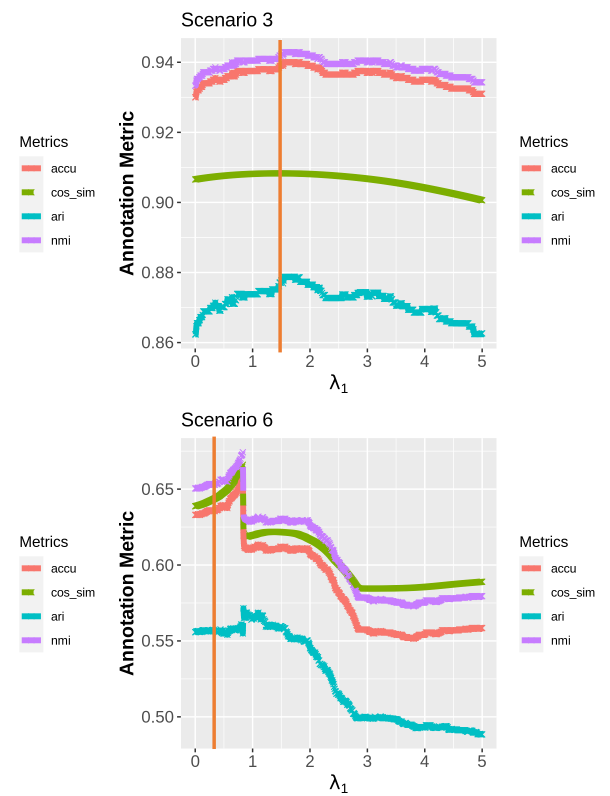

Fig3 


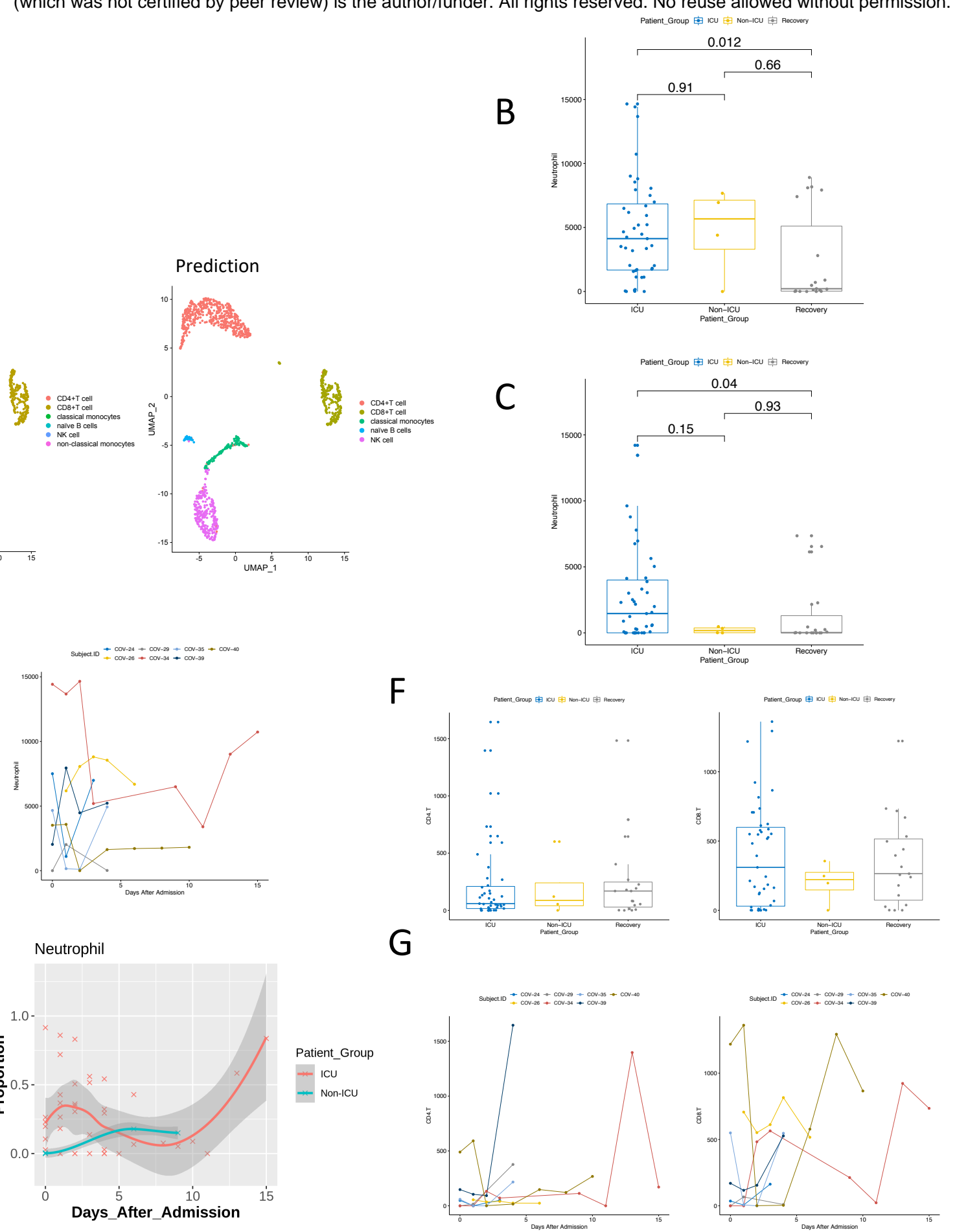



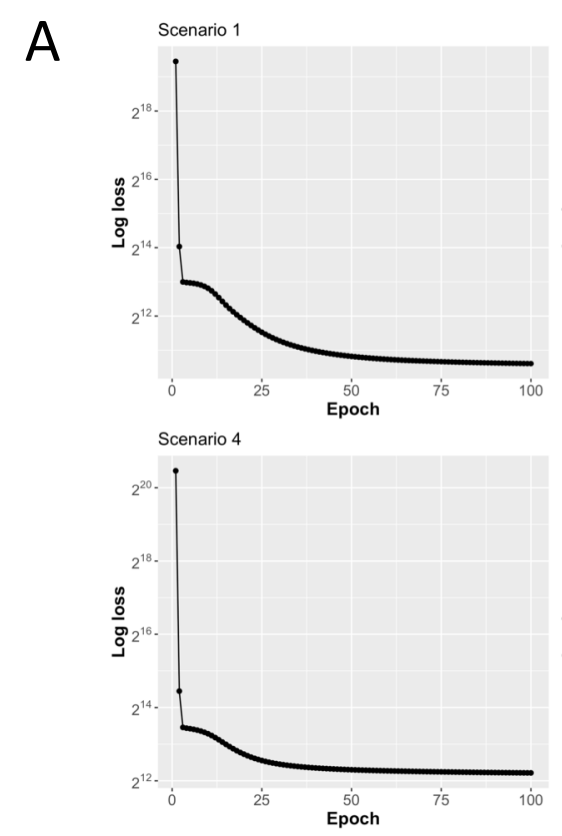

C
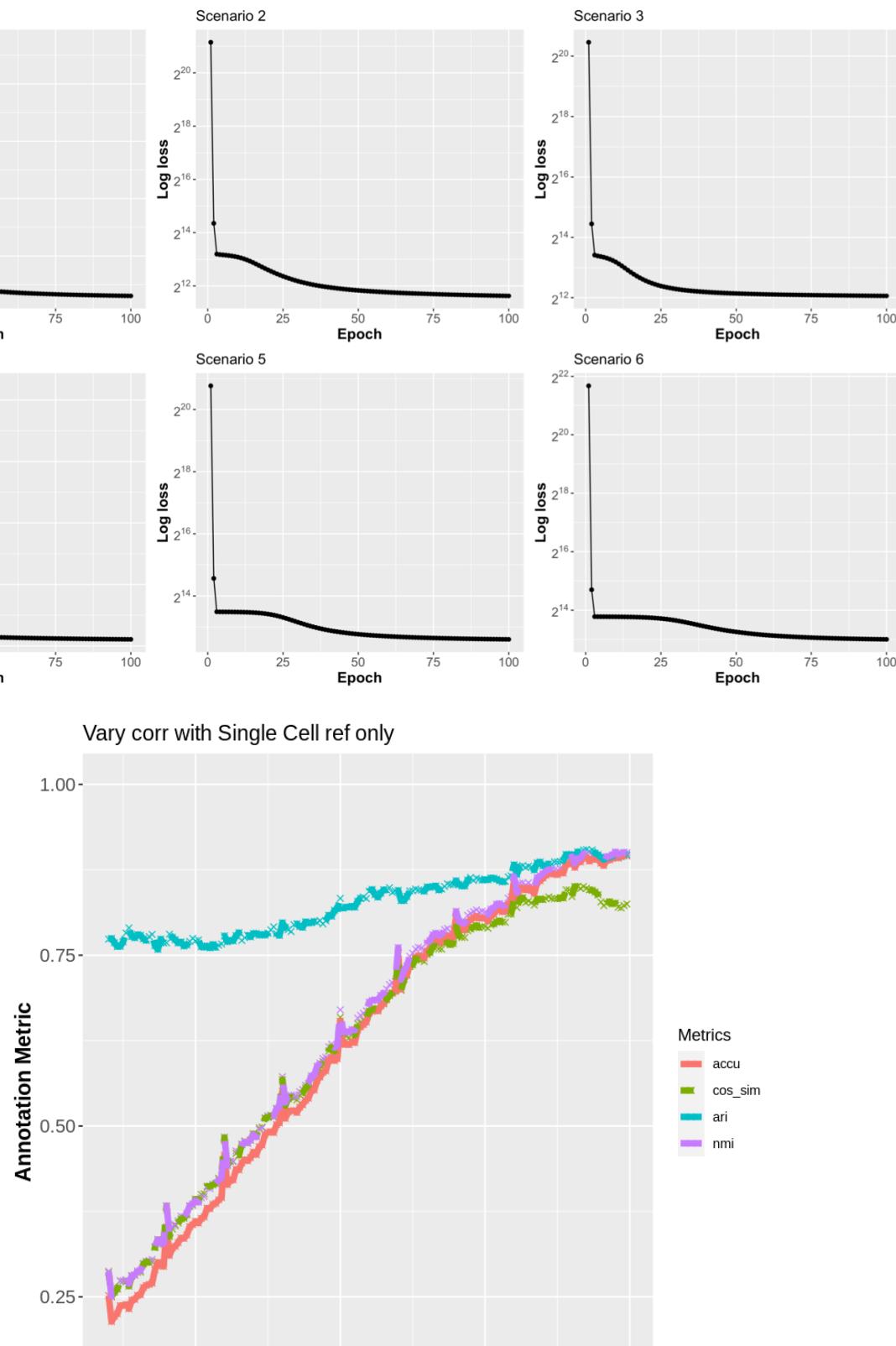

B
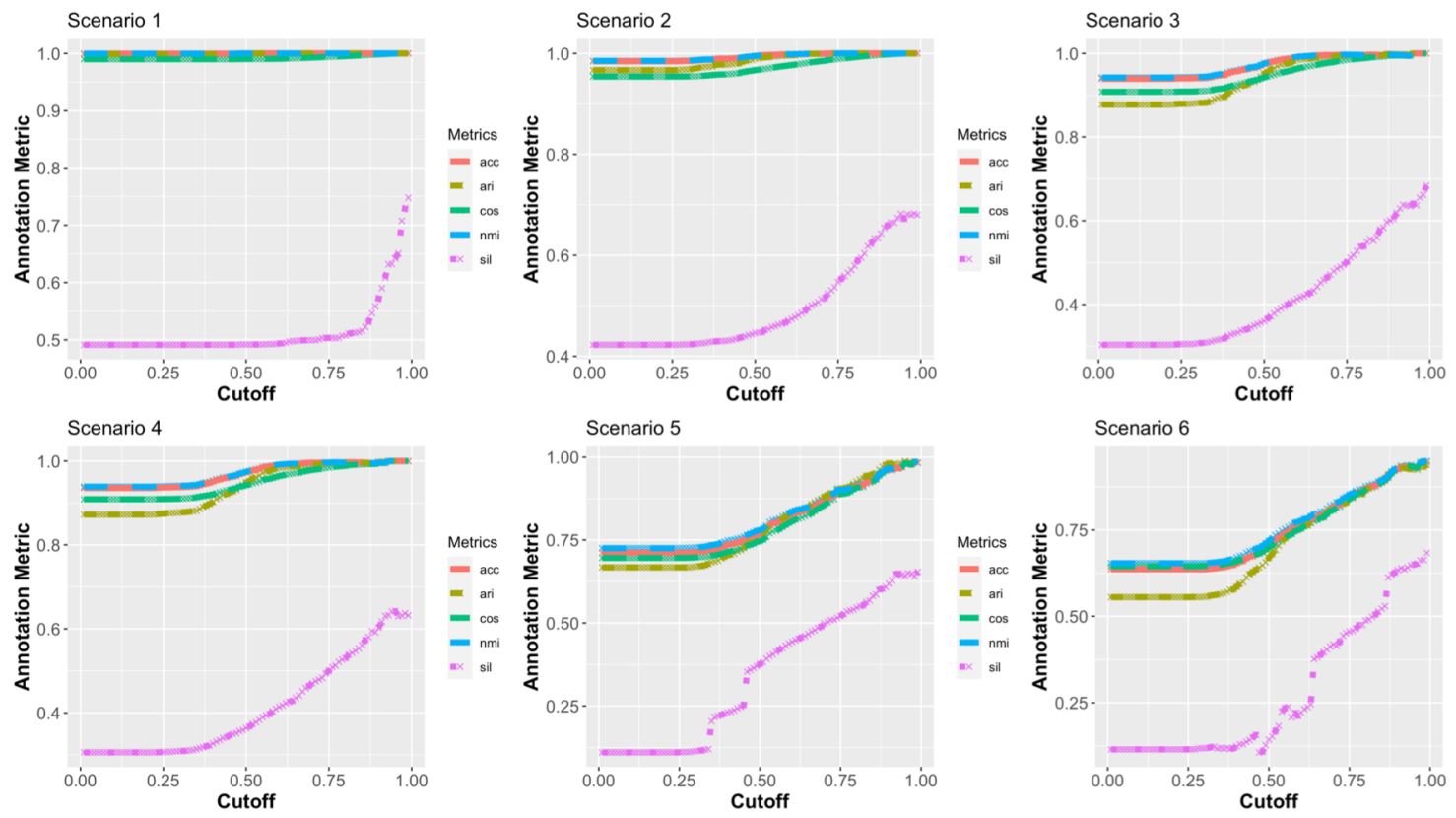

D
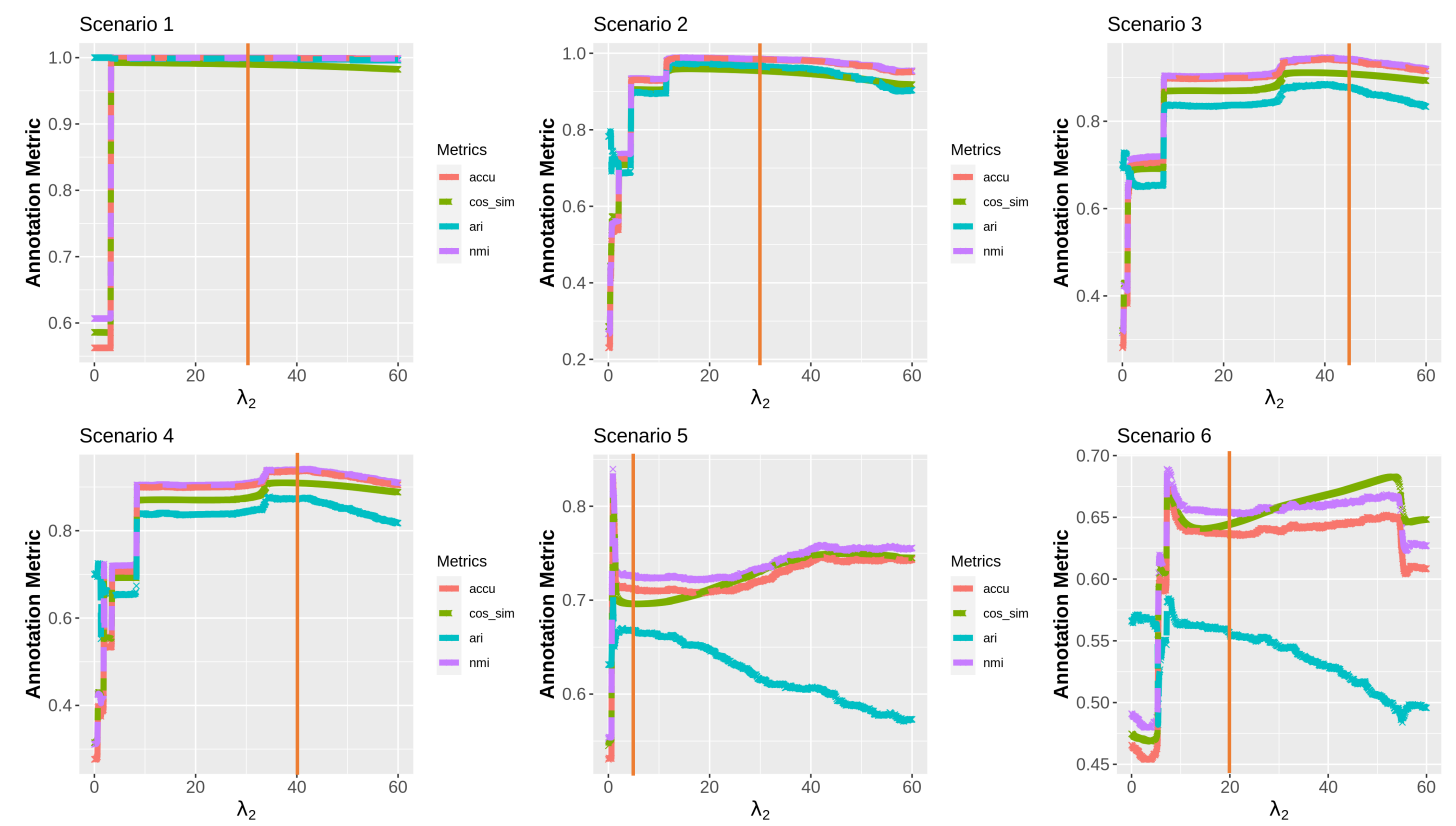
Scenario 1

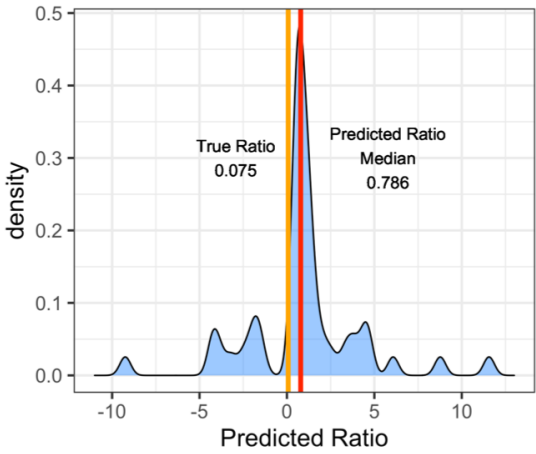

Scenario 4

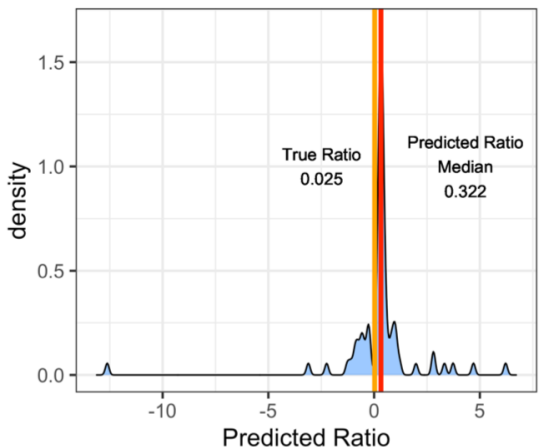

B

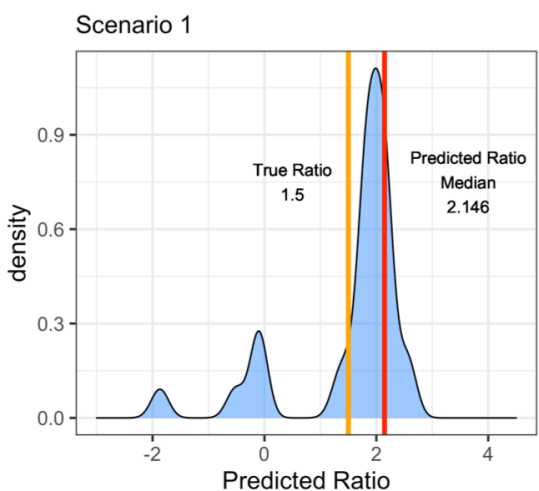

Scenario 4

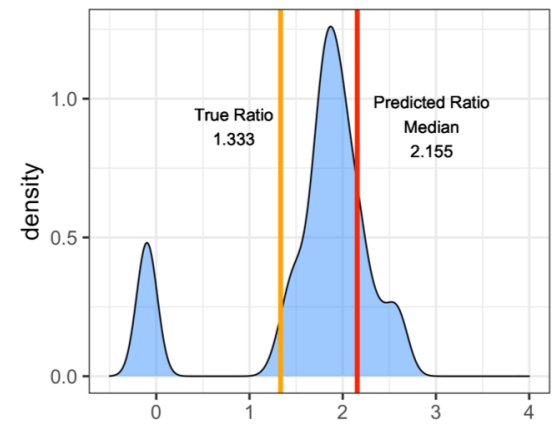

Supp2

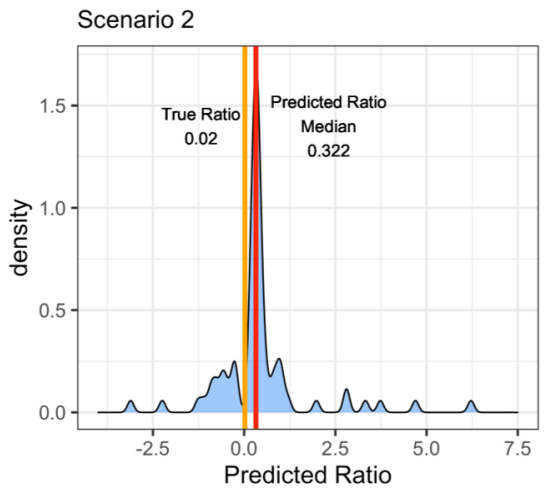

Scenario 5

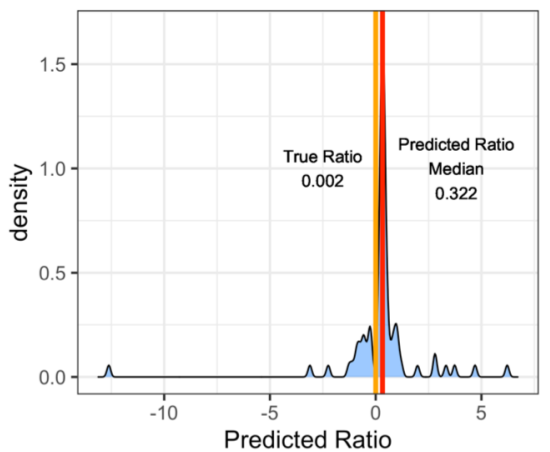

Ratio between predicted $\mathrm{W}$ and $\mathrm{A} 0$
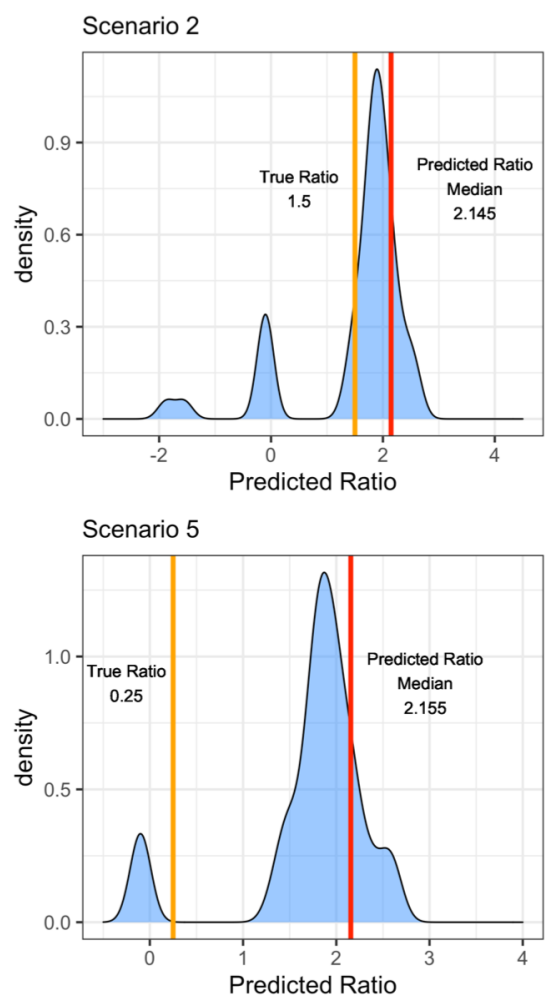

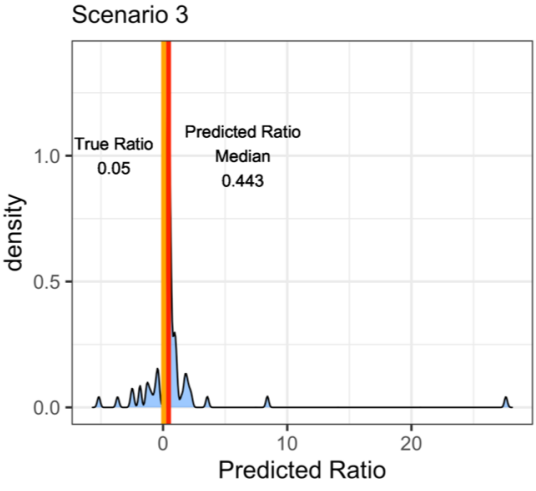

Scenario 6
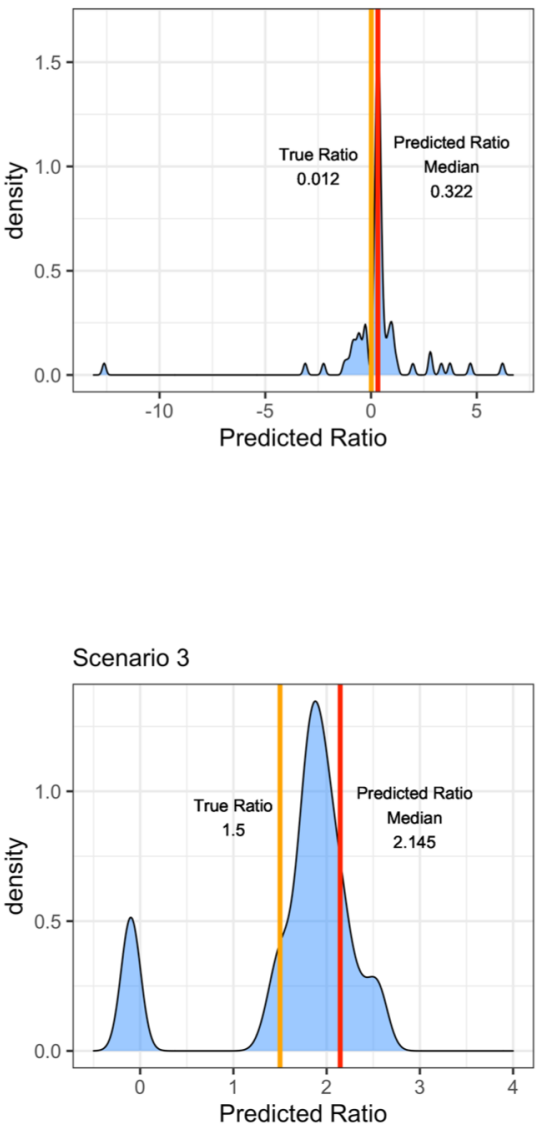

Scenario 6

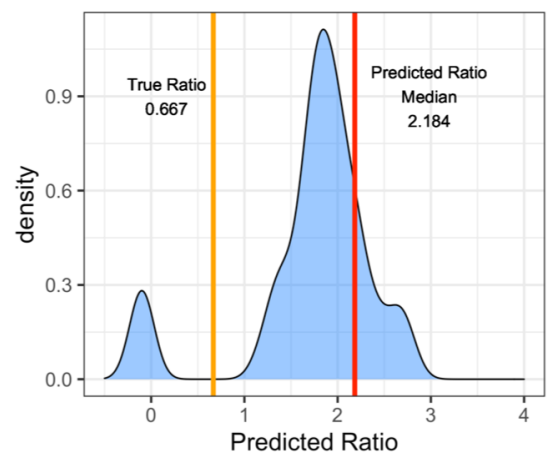


BCR-XLAccuracy 5 Cell types

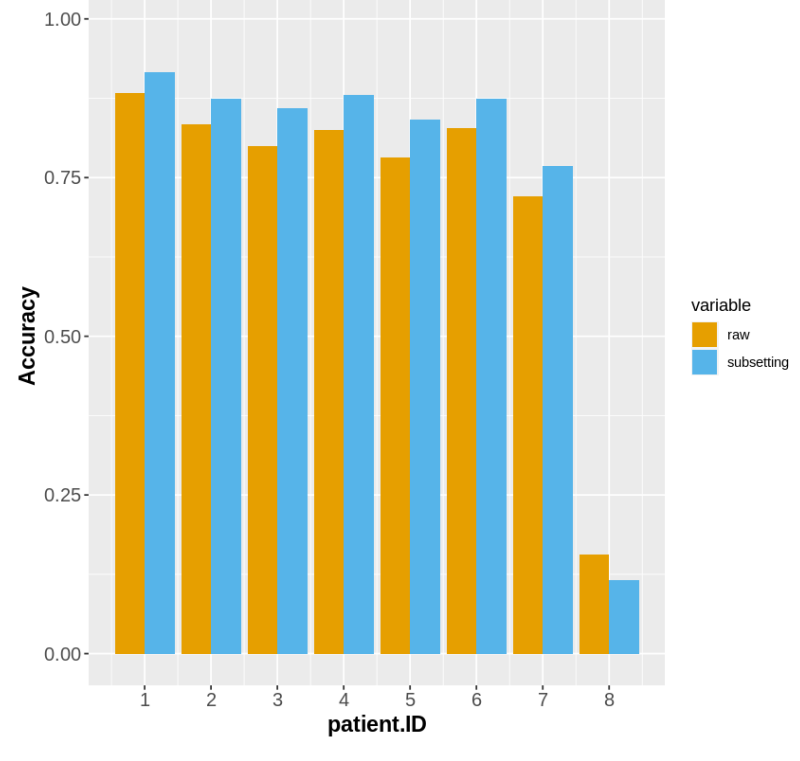

Reference Accuracy 5 Cell types

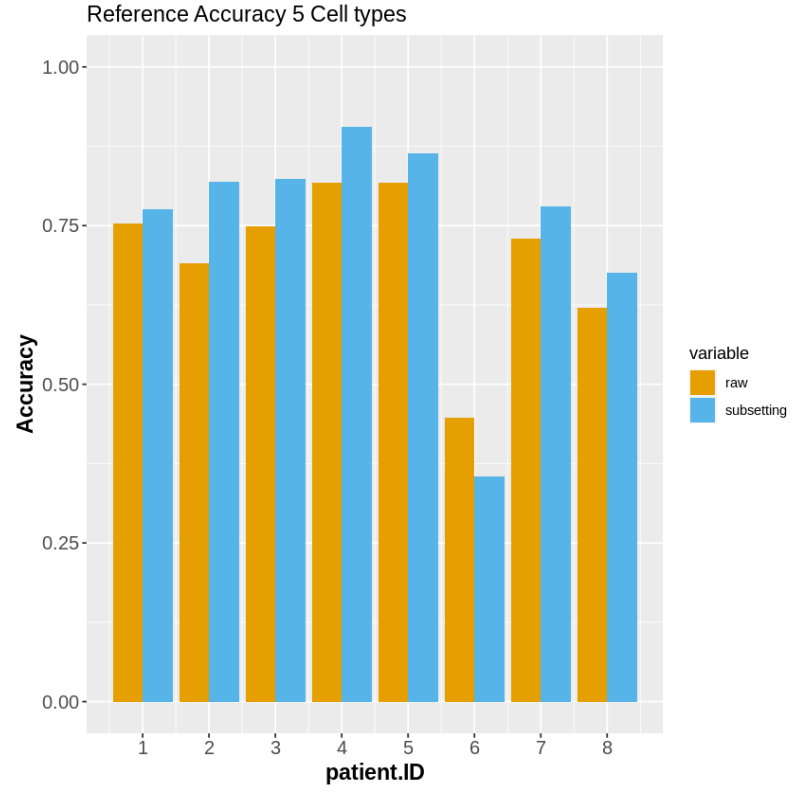

BCR-XLAccuracy 6 Cell types

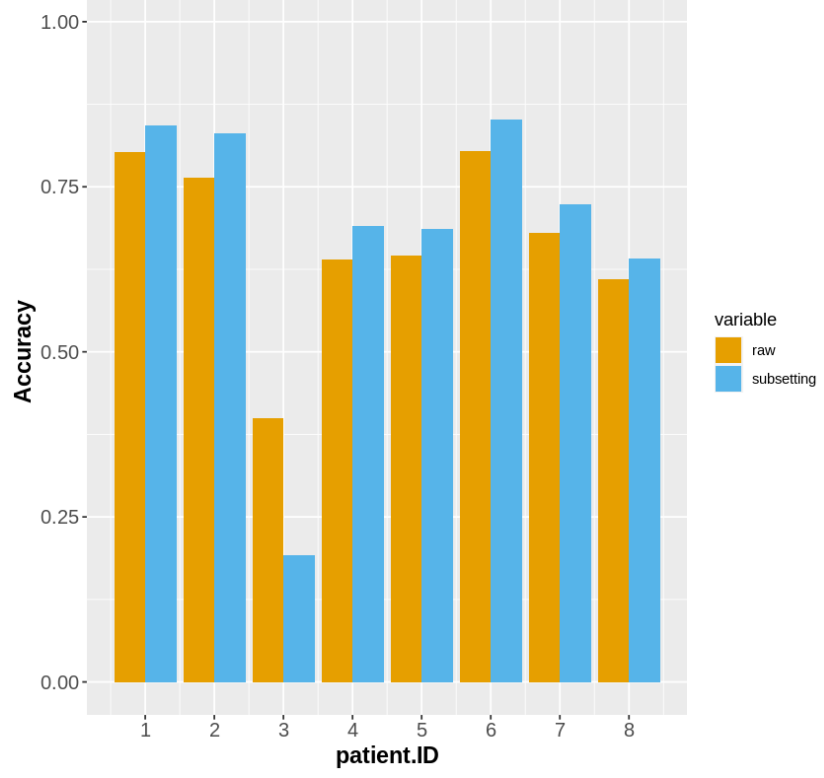

Reference Accuracy 6 Cell types

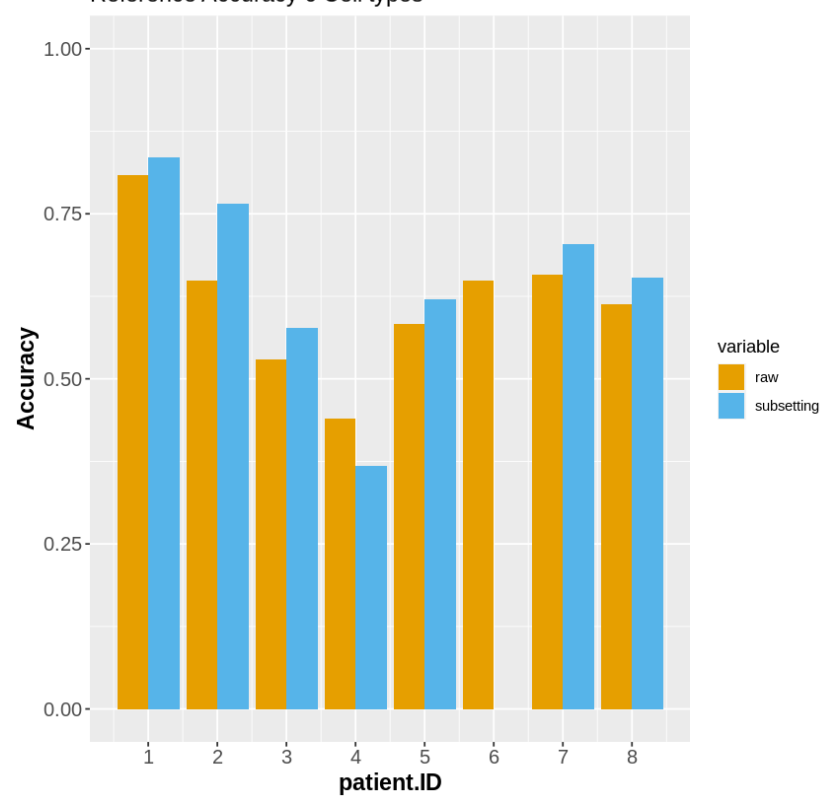

BCR-XLAccuracy 7 Cell types

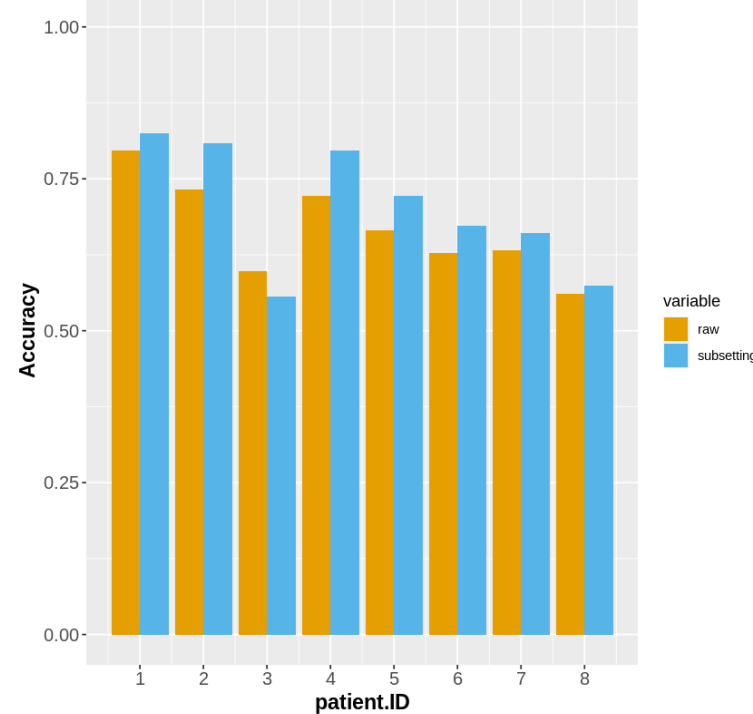

Reference Accuracy 7 Cell types

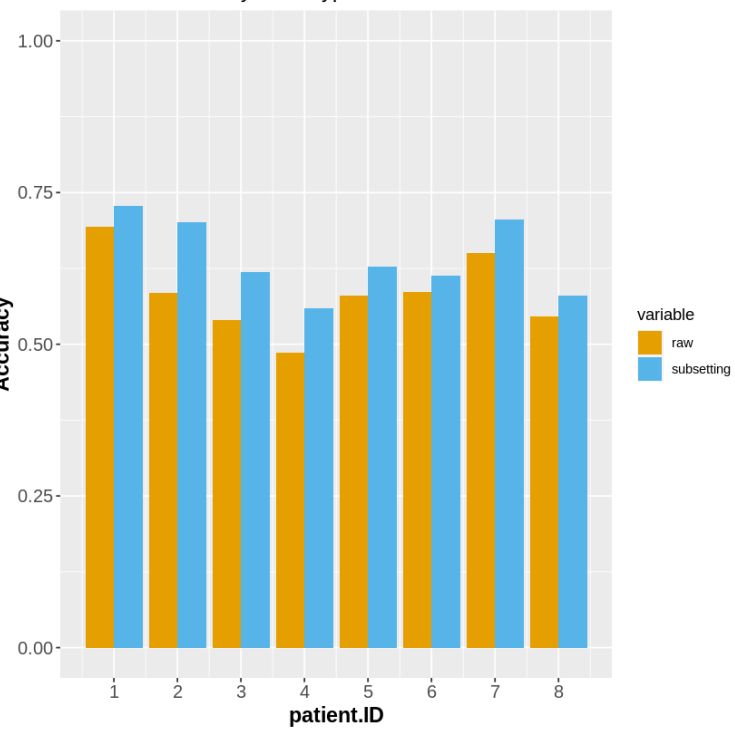

\section{Supp3}


A

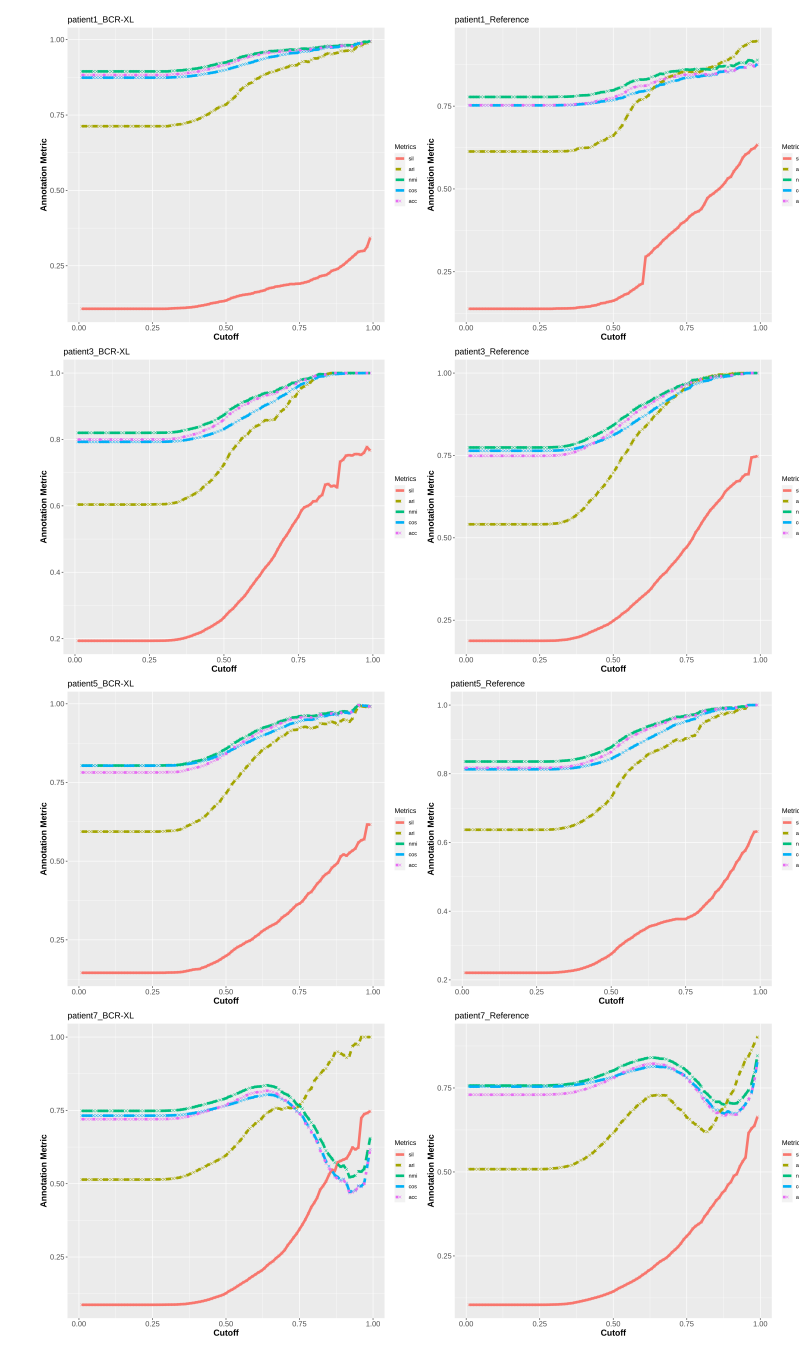

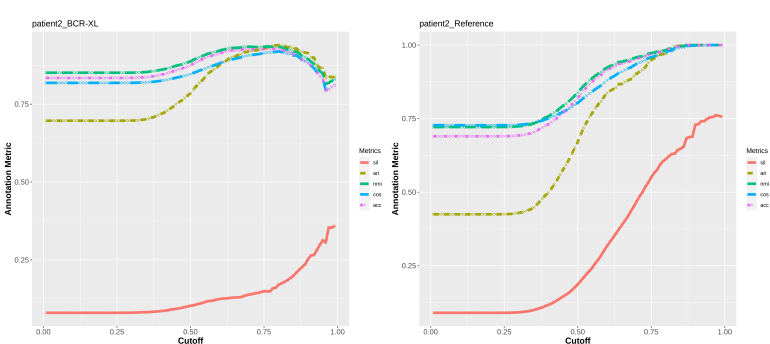
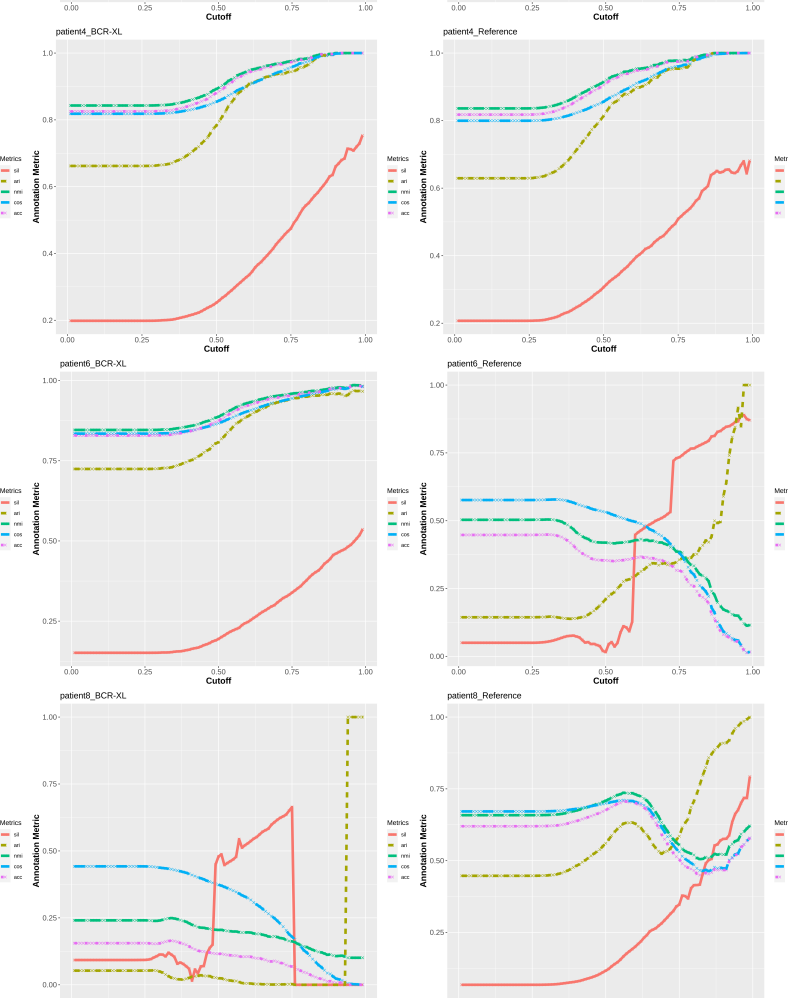

B

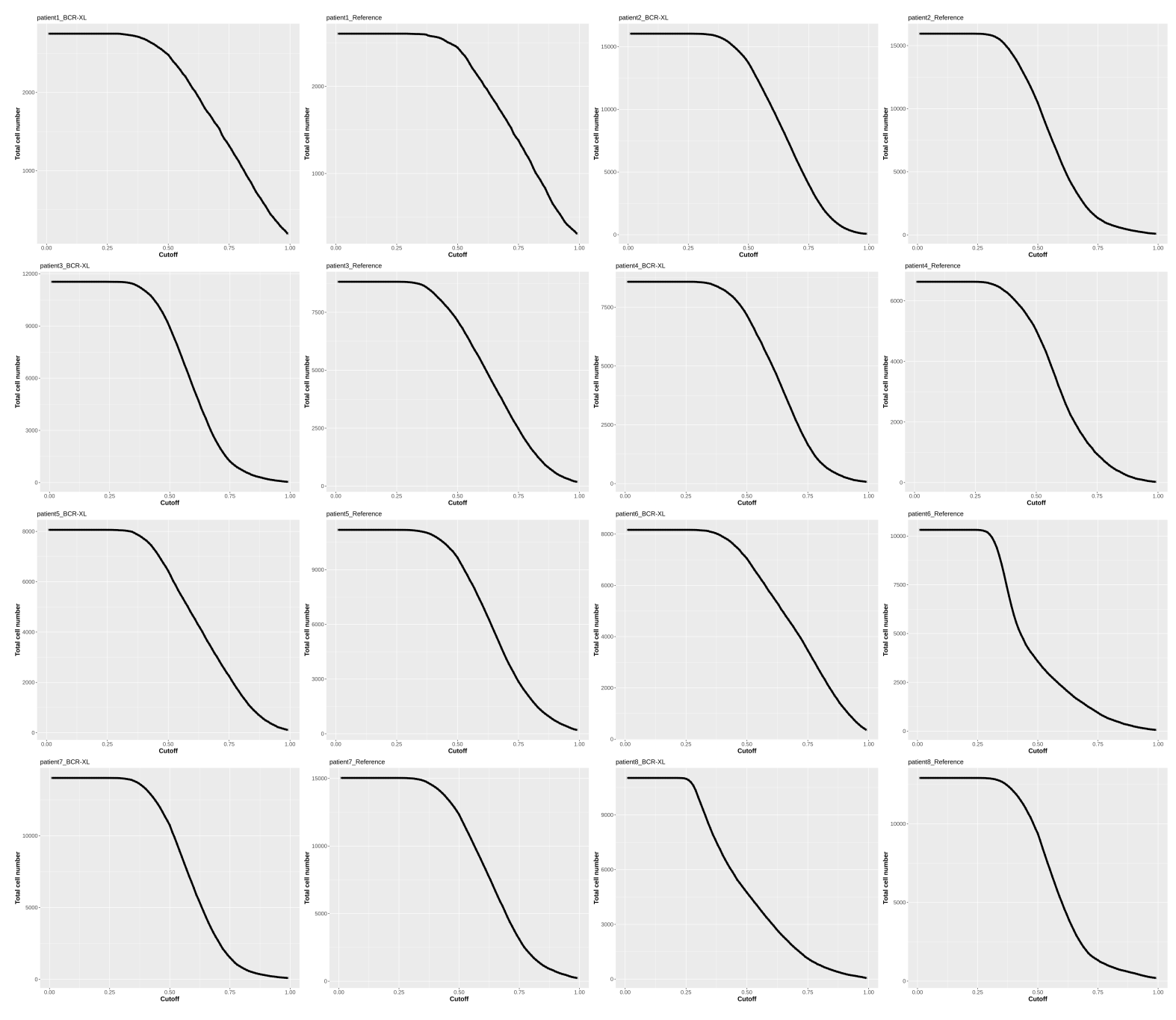

\section{Supp4}




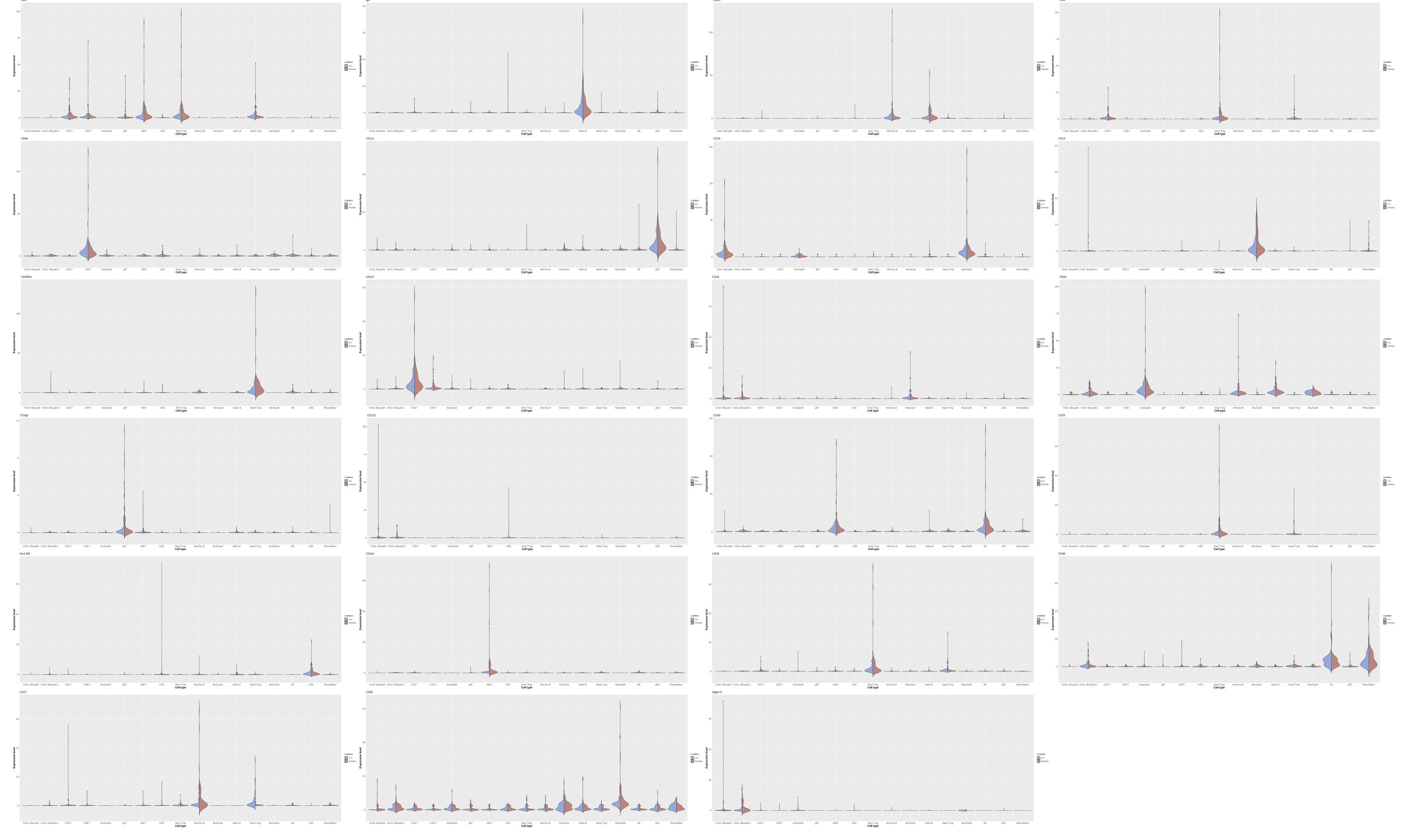

Supp5 


\begin{tabular}{|cccccccc|}
\hline Marker & memory B cells & naïve B cells & CD4 T-cells & CD8 T-cells & DC & monocytes & NK cells \\
CD3 & -1 & -1 & 1 & 1 & -1 & -1 & -1 \\
CD45 & 1 & 1 & 1 & 1 & 1 & 1 & 1 \\
CD4 & 0 & 0 & 1 & -1 & 0 & 0 & 0 \\
CD20 & 1 & 1 & 0 & 0 & -1 & -1 & -1 \\
CD33 & 0 & 0 & 0 & 0 & 0.5 & 1 & 0 \\
CD123 & 0 & 0 & 0 & 0 & 1 & 0 & 0 \\
CD14 & 0 & 0 & 0 & 0 & -1 & 1 & -1 \\
IgM & 1 & -1 & 0 & 0 & 0 & 0 & 0 \\
HLA-DR & 0 & 0 & 0 & 0 & 1 & 0 & -1 \\
CD7 & 0 & 0 & 1 & 1 & 0 & 0 & 1 \\
\hline
\end{tabular}




\begin{tabular}{|ccccccc|}
\hline Marker & CD4+T cell & CD8+T cells & naïve B cells & NK cell & $\begin{array}{c}\text { classical } \\
\text { monocytes }\end{array}$ & $\begin{array}{c}\text { non-classical } \\
\text { monocytes }\end{array}$ \\
CD154 & 0 & 0 & 0 & 0 & 1 & 1 \\
CD4 & 1 & -1 & 0 & 0 & 0 & 0 \\
CD56 & 0 & 0 & 0 & 1 & -1 & -1 \\
CD3 & 1 & 1 & -1 & -1 & -1 & -1 \\
CD19 & 0 & 0 & 1 & -1 & -1 & -1 \\
CD14 & 0 & 0 & 0 & -1 & 1 & -1 \\
CD11c & 0 & 0 & 0 & 1 & 0 & 0 \\
CD8 & -1 & 1 & 0 & 0 & 0 & 0 \\
CD16 & 0 & 0 & 0 & 1 & -1 & 1 \\
CD127 & 1 & 1 & 0 & 0 & 0 & 0 \\
\hline
\end{tabular}




\section{Table 3}

\begin{tabular}{|c|c|c|c|c|c|c|c|c|c|c|c|c|c|c|c|c|c|}
\hline marker & CD8 T & CD4 T & Mem Treg & Naive Treg & gdT & Naive B & Memory B & Plasmablast & NK & $\mathrm{pDC}$ & $\mathrm{mDC}$ & Monocyte & Eosinophil & Neutrophil & MAIT & CD16+ Basophil CD16- & - Basophil \\
\hline CD3 & 1 & 1 & 1 & 1 & 1 & -1 & -1 & -1 & -1 & -1 & -1 & -1 & -1 & -1 & 1 & -1 & -1 \\
\hline $\lg D$ & 0 & 0 & 0 & 0 & 0 & 1 & -1 & 0 & 0 & 0 & 0 & 0 & 0 & 0 & 0 & 0 & 0 \\
\hline CD20 & 0 & 0 & 0 & 0 & 0 & 1 & 1 & -1 & -1 & -1 & -1 & -1 & 0 & 0 & 0 & 0 & 0 \\
\hline CD4 & -1 & 1 & 1 & 1 & -1 & 0 & 0 & 0 & 0 & 0 & 0 & 0 & 0 & 0 & -1 & 0 & 0 \\
\hline CD8a & 1 & -1 & -1 & -1 & -1 & 0 & 0 & 0 & 0 & 0 & 0 & 0 & 0 & 0 & 0 & 0 & 0 \\
\hline CD11c & 0 & 0 & 0 & 0 & 0 & 0 & 0 & 0 & 0 & 1 & -1 & 0 & 0 & 0 & 0 & 0 & 0 \\
\hline CD14 & 0 & 0 & 0 & 0 & 0 & 0 & 0 & 0 & -1 & -1 & -1 & 1 & 0 & -1 & 0 & 0 & 0 \\
\hline CD45RA & 0 & 0 & -1 & 1 & 0 & 0 & 0 & 0 & 0 & 0 & 0 & 0 & 0 & 0 & 0 & 0 & 0 \\
\hline CD127 & 1 & 1 & -1 & -1 & 0 & 0 & 0 & 0 & 0 & 0 & 0 & 0 & 0 & 0 & 0 & 0 & 0 \\
\hline CD33 & 0 & 0 & 0 & 0 & 0 & 0 & 0 & 0 & 0 & 0 & 0 & 1 & 0 & 0 & 0 & 1 & 1 \\
\hline CD24 & 0 & 0 & 0 & 0 & 0 & 1 & 1 & 0 & 0 & 0 & 0 & 0 & 1 & 0 & 0 & 0 & 1 \\
\hline TCRgd & 0 & 0 & 0 & 0 & 1 & 0 & 0 & 0 & 0 & 0 & 0 & 0 & 0 & 0 & 0 & 0 & 0 \\
\hline CD123 & 0 & 0 & 0 & 0 & 0 & 0 & 0 & 0 & 0 & -1 & 1 & 0 & 0 & 0 & 0 & 1 & 1 \\
\hline CD25 & -1 & -1 & 1 & 1 & 0 & 0 & 0 & 0 & 0 & 0 & 0 & 0 & 0 & 0 & 0 & 0 & 0 \\
\hline HLA-DR & 0 & 0 & 0 & 0 & 0 & 0 & 0 & 0 & -1 & 1 & 1 & 0 & 0 & 0 & 0 & 0 & 0 \\
\hline CD161 & 0 & 0 & 0 & 0 & 0 & 0 & 0 & 0 & 0 & 0 & 0 & 0 & 0 & 0 & 1 & 0 & 0 \\
\hline CD28 & 0 & 0 & 1 & 1 & 0 & 0 & 0 & 0 & 0 & 0 & 0 & 0 & 0 & 0 & 0 & 0 & 0 \\
\hline CD38 & 0 & 0 & 0 & 0 & 0 & 0 & 0 & 1 & 1 & 0 & 0 & 0 & 0 & 0 & 0 & 0 & 0 \\
\hline CD27 & 0 & 0 & 0 & 0 & 0 & -1 & 1 & 0 & 0 & 0 & 0 & 0 & 0 & 0 & 0 & 0 & 0 \\
\hline CD55 & 0 & 0 & 0 & 0 & 0 & 0 & 0 & 0 & 0 & 0 & 0 & 0 & 0 & 1 & 0 & 0 & 0 \\
\hline Siglec-8 & 0 & 0 & 0 & 0 & 0 & 0 & 0 & 0 & 0 & 0 & 0 & 0 & 1 & 0 & 0 & 1 & 1 \\
\hline
\end{tabular}


Table 4

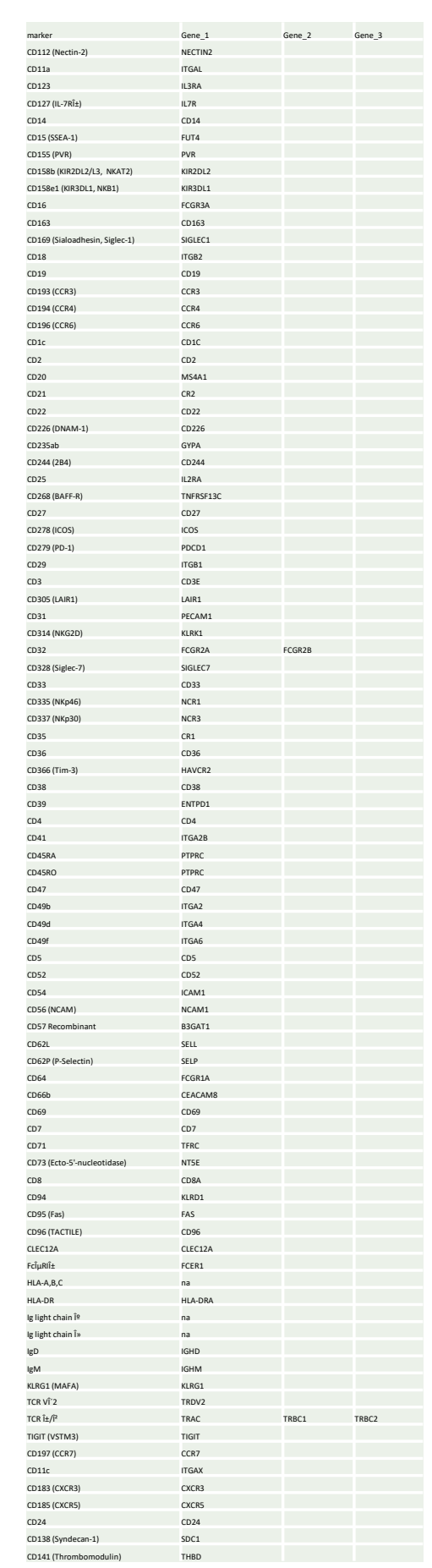




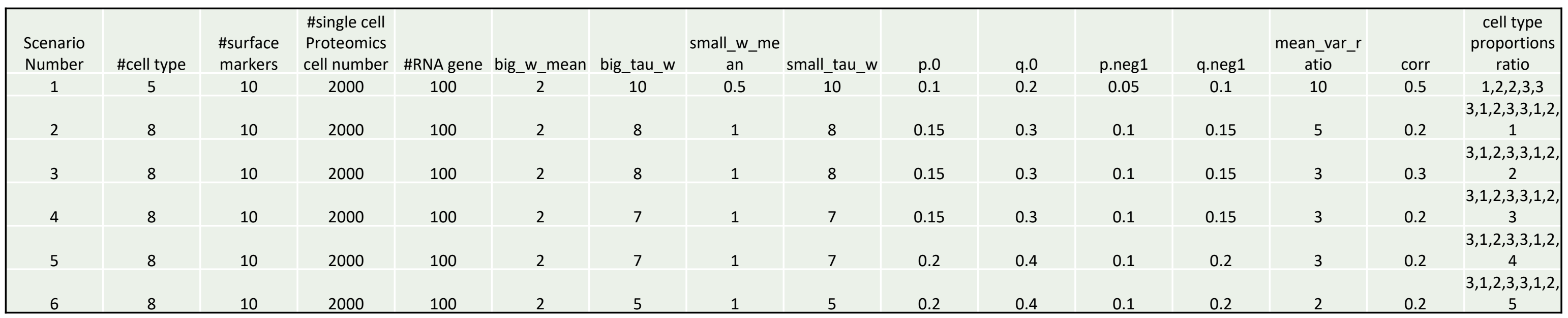




\section{Supplementary Materials}

\section{Parameter Tuning}

To get the optimal penalty parameters $\lambda_{1}, \lambda_{2}, \mu$ and $\eta$, we considered both the KarushKuhn-Tucker (KKT) condition combined with empirical screening. We first obtained the initial $W$ and $H$ by setting the arbitrary penalization: $\lambda_{1}=1, \lambda_{2}=10, \mu=50$ and $\eta=50$. This setting can give an acceptable result in most cases based on our empirical experiments. The first order derivatives of loss function are

$$
\begin{aligned}
& \nabla_{W} L(W, H)=W\left(H H^{T}+\mu I\right)-X H^{T}-\lambda_{1} A S-\lambda_{2} A_{0} \\
& \nabla_{H} L(W, H)=-W^{T} X+W^{T} W H+\eta 1_{K} 1_{K} 1_{K}^{T} H-\eta 1_{K} 1_{N}^{T}
\end{aligned}
$$

We first initialized $\eta$ by satisfying $\mathrm{KKT}$ conditions.

$$
\begin{array}{r}
\left(W\left(H H^{T}+\mu I\right)-X H^{T}-\lambda_{1} A S-\lambda_{2} A_{0}\right) \odot W=0 \\
\left(-W^{T} X+W^{T} W H+\eta 1_{K} 1_{K} 1_{K}^{T} H-\eta 1_{K} 1_{N}^{T}\right) \odot H=0 \\
W\left(H H^{T}+\mu I\right)-X H^{T}-\lambda_{1} A S-\lambda_{2} A_{0} \geq 0 \\
-W^{T} X+W^{T} W H+\eta 1_{K} 1_{K} 1_{K}^{T} H-\eta 1_{K} 1_{N}^{T} \geq 0
\end{array}
$$

by the second sufficient inequality, we set

$$
\eta:=\left\|\left(W^{T} W H-W^{T} X\right) /\left(1_{K} 1_{K}^{T} H-1_{K} 1_{N}^{T}\right)\right\|_{\text {median }}
$$

After getting the initial $\eta$, we initialized $\lambda 2$ and $\lambda_{1}$ in order by minimizing Adjusted Rank Index (ARI) with Louvain clustering. We considered the tuning parameters $\lambda 2$ and $\lambda_{1}$ from $0.1,1,10$, and 100 . The parameter $\mu$ is charging of the scale and penalization power on signature matrix $W$. Thus, a smaller $\mu$ can result in a larger norm of $W$. Therefore, we developed a new metric to evaluate the reliability by the difference between the mean value of expression profile $X$ and the mean value of signature matrix $W$. To eliminate the non-Gaussian effects, we also considered the difference between the median values of $X$ and $W$. Thus, the new metric is formulated as

$$
D(\mu):=\left(X_{\text {mean }}-W_{\text {mean }}\right)+\left(X_{\text {median }}-W_{\text {median }}\right)
$$

\section{Theoretical Proofs}

\section{Convergence of Algorithms}

Lemma 2.1. For any symmetric nonnegative matrix $Q \in \mathbb{R}^{K \times K}$ and row vector $w \in \mathbb{R}_{+}^{K}$ and $a \in \mathbb{R}_{\geq 0}^{K}$, the following matrix

$$
F=\operatorname{diag}\left\{\frac{(w Q+a)_{1}}{w_{1}}, \frac{(w Q+a)_{2}}{w_{2}}, \ldots, \frac{(w Q+a)_{K}}{w_{K}}\right\}-Q \in \mathbb{R}^{K \times K}
$$

is always semi-positive definite. 
Proof. We construct a new matrix $S \in \mathbb{R}^{K \times K}$ by $S_{i j}=w_{i} F_{i j} w_{j}$, where $S_{i j}$ is the element of $\mathrm{S}$ whose row is $i$ and column is $j$. And we reformulate $F$ to be

$$
F_{i j}= \begin{cases}-Q_{i j}, & \text { if } i \neq j \\ \frac{\Sigma_{k} w_{k} Q_{i k}+a_{i}}{w_{i}}-Q_{i i}, & \text { if } i=j\end{cases}
$$

For any nonnegative row vector $v \in \mathbb{R}^{K \times K}$, we have

$$
\begin{aligned}
v S v^{T} & =\Sigma_{i, j} v_{i} S_{i j} v_{j} \\
& =\Sigma_{i, j} v_{i} w_{i} F_{i j} w_{j} v_{j}
\end{aligned}
$$

Since row vectors $w$ and $v$ are nonnegative, $F$ is semi-positive definite when $S$ is semi-positive definite. Therefore, it is sufficient if we can prove $S$ is semi-positive definite. In the following, we follow equation 7 and prove that the product is always nonnegative.

$$
\begin{aligned}
v S v^{T} & =\Sigma_{i, j} v_{i} w_{i} F_{i j} w_{j} v_{j} \\
& =\Sigma_{i} v_{i} w_{i} \frac{\Sigma_{k} w_{k} Q_{i k}+a_{i}}{w_{i}} w_{i} v_{i}-\Sigma_{i, j} v_{i} w_{i} Q_{i j} w_{j} v_{j} \\
& =\Sigma_{i}\left(\Sigma_{k} w_{k} Q_{i k}\right) w_{i} v_{i}^{2}+\Sigma_{i} a_{i} w_{i} v_{i}^{2}-\Sigma_{i, j} v_{i} w_{i} Q_{i j} w_{j} v_{j} \\
& =\Sigma_{i, j} w_{i} w_{j} Q_{i j} v_{i}^{2}+\Sigma_{i} a_{i} w_{i} v_{i}^{2}-\Sigma_{i, j} w_{i} w_{j} Q_{i j} v_{i} v_{j} \\
& =\Sigma_{i, j} w_{i} w_{j} Q_{i j}\left(v_{i}^{2}-v_{i} v_{j}\right)+\Sigma_{i} a_{i} w_{i} v_{i}^{2} \\
& =\frac{1}{2} \Sigma_{i, j} w_{i} w_{j} Q_{i j}\left(v_{i}^{2}+v_{j}^{2}-2 v_{i} v_{j}\right)+\Sigma_{i} a_{i} w_{i} v_{i}^{2} \\
& =\frac{1}{2} \Sigma_{i, j}\left|v_{i}-v_{j}\right| w_{i} Q_{i j} w_{j}\left|v_{i}-v_{j}\right|+\Sigma_{i} a_{i} w_{i} v_{i}^{2} \geq 0
\end{aligned}
$$

The first term in the above formula is nonnegative since $w$ is nonnegative and $Q$ is semi-positive definite. The second term is always greater than or equal to 0 due the nonnegativity of $a$ and $w$. Thus, $S$ is a semi-positive definite matrix.

Theorem 2.2. Consider the following quadratic optimization problem,

$$
\min _{w \geq 0} E(w)=\frac{1}{2}\|x-w H\|_{F}^{2}-\lambda_{1} \operatorname{tr}\left(w^{T} a_{1}\right)-\lambda_{2} \operatorname{tr}\left(w^{T} a_{2}\right)+\frac{\mu}{2}\|w\|^{2}
$$

which is the loss function for a row vector $w \in \mathbb{R}^{K}$. In this optimization problem, $H \in \mathbb{R}^{K \times N}$ is a constant non-negative matrix, and $x, a_{1}, a_{2} \in \mathbb{R}^{K}$ are constant row vectors. The penalty parameters, $\lambda_{1}, \lambda_{2}$, and $\mu$ are positive numbers. The the following update rule

$$
w_{j}^{t+1}=w_{j}^{t} \frac{\left[x H^{T}\right]_{j}^{+}+\lambda_{1}\left[(A S)_{i}\right]_{j}^{+}+\lambda_{2}\left[\left(A_{0}\right)_{i}\right]_{j}^{+}}{\left[w_{i}\left(H H^{T}+\mu I\right)\right]_{j}+\left[x H^{T}\right]_{j}^{-}+\lambda_{1}\left[a_{1}\right]_{j}^{-}+\lambda_{2}\left[a_{2}\right]_{j}^{-}}
$$

where we denote

$$
\begin{aligned}
& x^{+}= \begin{cases}x, & \text { if } x \geq 0 \\
0, & \text { if } x<0\end{cases} \\
& x^{-}= \begin{cases}0, & \text { if } x>0 \\
-x, & \text { if } x \leq 0\end{cases}
\end{aligned}
$$

converges to its optimal solution with the convergence rate

$$
\min _{t \in[1, K]}\left\|w^{t+1}-w^{t}\right\| \leq \frac{2}{\mu(K-1)} E\left(w^{0}\right)
$$


Proof. To prove that there exists a $w^{t+1}$ that $E\left(w^{t+1}\right) \geq E\left(w^{t}\right)$, we would like to construct an auxiliary function $F\left(w, w^{t}\right)$, s.t.

$$
\begin{aligned}
F\left(w^{t}, w^{t}\right) & =E\left(w^{t}\right) \\
F\left(w, w^{t}\right) & \geq E(w)
\end{aligned}
$$

Then we have

$$
E\left(w^{t}\right)=F\left(w^{t}, w^{t}\right) \geq F\left(w^{t+1}, w^{t}\right) \geq E\left(w^{t+1}\right)
$$

where $w^{t+1}=\arg \min _{w} F\left(w, w^{t}\right)$. Thus, the loss function $E\left(w^{t}\right)$ is monotonously non-increasing w.r.t the iteration $t$. We define the auxiliary function as the following.

$$
F\left(w, w^{t}\right)=E\left(w^{t}\right)+\nabla E\left(w^{t}\right)\left(w-w^{t}\right)^{T}+\frac{1}{2}\left(w-w^{t}\right) J\left(w^{t}\right)\left(w-w^{t}\right)^{T}
$$

where

$$
\begin{array}{r}
J\left(w^{t}\right)=\quad \operatorname{diag}\left(\frac{w_{1}^{t}\left[H H^{T}+\mu I\right]_{1}+\left[x H^{T}\right]_{1}^{-}+\left[\lambda_{1} a_{1}\right]_{1}^{-}+\left[\lambda_{2} a_{2}\right]_{1}^{-}}{w_{1}^{t}},\right. \\
\frac{w_{2}^{t}\left[H H^{T}+\mu I\right]_{2}+\left[x H^{T}\right]_{2}^{-}+\left[\lambda_{1} a_{1}\right]_{2}^{-}+\left[\lambda_{2} a_{2}\right]_{2}^{-}}{w_{2}^{t}}, \\
\left.\ldots, \frac{w_{K}^{t}\left[H H^{T}+\mu I\right]_{K}+\left[x H^{T}\right]_{K}^{-}+\left[\lambda_{1} a_{1}\right]_{K}^{-}+\left[\lambda_{2} a_{2}\right]_{K}^{-}}{w_{K}^{t}}\right)
\end{array}
$$

We can approximate $E\left(w^{t}\right)$ based on Taylor expansion.

$$
E(w)=E\left(w^{t}\right)+\nabla E\left(w^{t}\right)\left(w-w^{t}\right)^{T}+\frac{1}{2}\left(w-w^{t}\right) \nabla^{2} E\left(w^{t}\right)\left(w-w^{t}\right)^{T}
$$

where, by simple derivation,

$$
\begin{aligned}
\nabla E\left(w^{t}\right) & =w^{t}\left[H H^{T}+\mu I\right]+\left[x H^{T}\right]^{-}+\left[\lambda_{1} a_{1}\right]^{-}+\left[\lambda_{2} a_{2}\right]^{-}-\left[x H^{T}\right]^{+}-\left[\lambda_{1} a_{1}\right]^{+}-\left[\lambda_{2} a_{2}\right]^{+} \\
\nabla^{2} E\left(w^{t}\right) & =H H^{T}+\mu I
\end{aligned}
$$

Now we can have 14 satisfied if $F\left(w^{t+1}, w^{t}\right) \geq E\left(w^{t+1}\right)$. To get it, we have

$$
F\left(w^{t+1}, w^{t}\right)-E\left(w^{t+1}\right)=\frac{1}{2}\left(w-w^{t}\right)\left[J\left(w^{t}\right)-\nabla^{2} E\left(w^{t}\right)\right]\left(w-w^{t}\right)^{T}
$$

Obviously, $\nabla^{2} E\left(w^{t}\right)=H H^{T}+\mu I$ is a positive definite matrix since $\mu$ is positive. Furthermore, the row vector

$$
\left(x H^{T}\right)^{-}+\left[\lambda_{1} a_{1}\right]^{-}+\left[\lambda_{2} a_{2}\right]^{-}
$$

is always nonnegative. Due to the 2.1. $J\left(w^{t}\right)-\nabla^{2} E\left(w^{t}\right)$ is a semi-positive definite matrix. So that $F\left(w^{t+1}, w^{t}\right) \geq E\left(w^{t+1}\right)$ is always satisfied and $E\left(w^{t}\right)$ is nonincreasing w.r.t to $t$. Next, we would like to find the optimizer of $F\left(w, w^{t}\right)$. To achieve this, we set $\left.\frac{\partial F\left(w, w^{t}\right)}{\partial w}\right|_{w=w^{t+1}}=0$, which is equivalent to equation $\nabla E\left(w^{t}\right)+\left(w^{t+1}-w^{t}\right) J\left(w^{t}\right)=0$. Therefore, we have

$$
w^{t+1}=w^{t}-J^{-1}\left(w^{t}\right) \nabla E\left(w^{t}\right)
$$

For each element of $w^{t+1}$, the above formula can be reformulated as

$$
\begin{aligned}
w_{j}^{t+1} & =w_{j}^{t}-J^{-1}\left(w^{t}\right)_{j} \nabla E\left(w^{t}\right)_{j} \\
& =w_{j}^{t}-\frac{w_{j}^{t}}{w_{j}^{t}\left[H H^{T}+\mu I\right]_{j}+\left[x H^{T}\right]_{j}^{-}+\left[\lambda_{1} a_{1}\right]_{j}^{-}+\left[\lambda_{2} a_{2}\right]_{j}^{-}} \\
& \cdot\left[w^{t}\left[H H^{T}+\mu I\right]+\left[x H^{T}\right]^{-}+\left[\lambda_{1} a_{1}\right]^{-}+\left[\lambda_{2} a_{2}\right]^{-}-\left[x H^{T}\right]^{+}-\left[\lambda_{1} a_{1}\right]^{+}-\left[\lambda_{2} a_{2}\right]^{+}\right] \\
& =w_{j}^{t} \frac{\left[x H^{T}\right]_{j}^{+}+\lambda_{1}\left[(A S)_{i}\right]_{j}^{+}+\lambda_{2}\left[\left(A_{0}\right)_{i}\right]_{j}^{+}}{\left[w_{i}\left(H H^{T}+\mu I\right)\right]_{j}+\left[x H^{T}\right]_{j}^{-}+\lambda_{1}\left[a_{1}\right]_{j}^{-}+\lambda_{2}\left[a_{2}\right]_{j}^{-}}
\end{aligned}
$$


So far we have proved that the above updating rule can make $E\left(w^{t}\right)$ nonincreasing w.r.t to the $t$. Specifically, we have the lower bound for every element on the diagonal of $J$ matrix.

$$
J\left(w^{t}\right)_{j j}=\frac{w_{j}^{t}\left[H H^{T}+\mu I\right]_{j}+\left[x H^{T}\right]_{j}^{-}+\left[\lambda_{1} a_{1}\right]_{j}^{-}+\left[\lambda_{2} a_{2}\right]_{j}^{-}}{w_{j}^{t}} \geq \mu
$$

Based on 14 and 23 , we can derive the lower bound of the difference between two loss function values after one update.

$$
\begin{aligned}
E\left(w^{t}\right)-E\left(w^{t+1}\right) & \geq F\left(w^{t}, w^{t}\right)-F\left(w^{t+1}, w^{t}\right) \\
& =\nabla F\left(w^{t+1}, w^{t}\right)\left(w^{t+1}-w^{t}\right)+\frac{1}{2}\left(w^{t}-w^{t+1}\right) \nabla^{2} F\left(w^{t+1}, w^{t}\right)\left(w^{t}-w^{t+1}\right)^{T} \\
& =\frac{1}{2}\left(w^{t}-w^{t+1}\right) J\left(w^{t}\right)\left(w^{t}-w^{t+1}\right)^{T} \\
& \geq \frac{\mu}{2}\left\|w^{t}-w^{t+1}\right\|^{2}
\end{aligned}
$$

since $\nabla F\left(w^{t+1}, w^{t}\right)=0$ and $\nabla^{2} F\left(w^{t+1}, w^{t}\right)=J\left(w^{t}\right)$. And by accumulating $\left\|w^{t}-w^{t+1}\right\|^{2}$ from 0 to $T-1$, we then can have

$$
E\left(w^{0}\right)-E\left(w^{K}\right) \geq \frac{\mu}{2} \Sigma_{t=0}^{T-1}\left\|w^{t}-w^{t+1}\right\|^{2}
$$

Thus, for any given fixed iteration number $T$, we can have

$$
\min _{0 \leq t \leq T}\left\|w^{t}-w^{t+1}\right\|^{2} \leq \frac{2}{\mu(T-1)}\left(E\left(w^{0}\right)-E\left(w^{T}\right)\right) \leq \frac{2}{\mu(T-1)} E\left(w^{0}\right)
$$

Theorem 2.3. Consider the following quadratic optimization problem,

$$
\min _{w \geq 0} E(h)=\frac{1}{2}\|x-W h\|_{F}^{2}+\frac{\eta}{2}\left\|1_{k}^{T} h-1_{N}^{T}\right\|_{2}^{2}
$$

which is the loss function for a column vector $h \in \mathbb{R}^{K}$. In this optimization problem, $W \in \mathbb{R}^{D \times K}$ is the constant non-negative matrix, and $x \in \mathbb{R}^{K}$ is a constant column vectors. The penalty parameter, $\eta$ is a positive number. The the following update rule

$$
h_{j}^{t+1}=h_{j}^{t} \frac{\left[W^{T} x\right]_{j}^{+}+\eta 1_{k}}{\left[\left(W^{T} W+\eta 1_{k} 1_{k}^{T}\right) h^{t}\right]_{j}+\left[W^{T} x\right]_{j}^{-}}
$$

converges to its optimal solution with the convergence rate

$$
\min _{t \in[1, K]}\left\|h^{t+1}-h^{t}\right\| \leq \frac{2}{\eta(K-1)} E\left(h^{0}\right)
$$

Proof. To prove that there exists an $h^{t+1}$ that $E\left(h^{t+1}\right) \geq E\left(h^{t}\right)$, we can construct an auxiliary function $F\left(h, h^{t}\right)$, s.t.

$$
\begin{gathered}
F\left(h^{t}, h^{t}\right)=E\left(h^{t}\right) \\
F\left(h, h^{t}\right) \geq E(h)
\end{gathered}
$$

Then we have

$$
E\left(h^{t}\right)=F\left(h^{t}, h^{t}\right) \geq F\left(h^{t+1}, h^{t}\right) \geq E\left(h^{t+1}\right)
$$

where $h^{t+1}=\arg \min _{h} F\left(h, h^{t}\right)$. Thus, the loss function $E\left(h^{t}\right)$ is monotonously non-increasing w.r.t the iteration $t$. We define the auxiliary function as the following.

$$
F\left(h, h^{t}\right)=E\left(h^{t}\right)+\left(h-h^{t}\right)^{T} \nabla E\left(h^{t}\right)+\frac{1}{2}\left(h-h^{t}\right)^{T} J\left(h^{t}\right)\left(h-h^{t}\right)
$$


where

$$
\begin{array}{r}
J\left(h^{t}\right)=\operatorname{diag}\left(\frac{\left[\left(W^{T} W+\eta 1_{K} 1_{K}^{T}\right) h^{t}\right]_{1}+\left[W^{T} x\right]_{1}^{-}}{h_{1}^{t}},\right. \\
\frac{\left[\left(W^{T} W+\eta 1_{K} 1_{K}^{T}\right) h^{t}\right]_{2}+\left[W^{T} x\right]_{2}^{-}}{h_{2}^{t}}, \\
\left.\ldots, \frac{\left[\left(W^{T} W+\eta 1_{K} 1_{K}^{T}\right) h^{t}\right]_{K}+\left[W^{T} x\right]_{K}^{-}}{h_{K}^{t}}\right)
\end{array}
$$

We can approximate $E\left(h^{t}\right)$ based on Taylor expansion.

$$
E(h)=E\left(h^{t}\right)+\left(h-h^{t}\right)^{T} \nabla E\left(h^{t}\right)+\frac{1}{2}\left(h-h^{t}\right)^{T} \nabla^{2} E\left(h^{t}\right)\left(h-h^{t}\right)
$$

where, by simple derivation,

$$
\begin{aligned}
\nabla E\left(h^{t} ; W\right) & =\left(W^{T} W+\eta 1_{k} 1_{k}^{T}\right) h^{t}+\left[W^{T} x\right]^{-}-\left[W^{T} x\right]^{+}-\eta 1_{K} \\
\nabla^{2} E\left(h^{t}\right) & =W^{T} W+\eta 1_{k} 1_{k}^{T}
\end{aligned}
$$

Now we can have 31 satisfied if $F\left(h^{t+1}, h^{t}\right) \geq E\left(h^{t+1}\right)$. To get it, we have

$$
F\left(h^{t+1}, h^{t}\right)-E\left(h^{t+1}\right)=\frac{1}{2}\left(h-h^{t}\right)^{T}\left[J\left(w^{t}\right)-\nabla^{2} E\left(h^{t}\right)\right]\left(h-h^{t}\right)
$$

Obviously, $\nabla^{2} E\left(h^{t}\right)=W^{T} W+\eta 1_{k} 1_{k}^{T}$ is a positive definite matrix since $\eta$ is positive. Furthermore, the row vector

$$
\left(W^{T} x\right)^{-}
$$

is always nonnegative. Due to the 2.1. $J\left(h^{t}\right)-\nabla^{2} E\left(h^{t}\right)$ is a semi-positive definite matrix. So that $F\left(h^{t+1}, h^{t}\right) \geq E\left(h^{t+1}\right)$ is always satisfied and $E\left(h^{t}\right)$ is nonincreasing w.r.t to $t$. Nextly, we would like to find the optimizer of $F\left(h, h^{t}\right)$. To achieve this, we set $\left.\frac{\partial F\left(h, h^{t}\right)}{\partial h}\right|_{h=h^{t+1}}=0$, which is equivalent to equation $\nabla E\left(h^{t}\right)+\left(h^{t+1}-h^{t}\right) J\left(h^{t}\right)=0$. Therefore, we have

$$
h^{t+1}=h^{t}-J^{-1}\left(h^{t}\right) \nabla E\left(h^{t}\right)
$$

For each element of $h^{t+1}$, the above formula can be reformulated as

$$
\begin{aligned}
h_{j}^{t+1} & =h_{j}^{t}-J^{-1}\left(h^{t}\right)_{j} \nabla E\left(h^{t}\right)_{j} \\
& =h_{j}^{t}-\frac{h_{j}^{t}}{\left[\left(W^{T} W+\eta 1_{K} 1_{K}^{T}\right) h^{t}\right]_{j}+\left[W^{T} x\right]_{j}^{-}} \\
& \cdot\left[\left(W^{T} W+\eta 1_{k} 1_{k}^{T}\right) h^{t}+\left[W^{T} x\right]^{-}-\left[W^{T} x\right]^{+}-\eta 1_{K}\right] \\
& =h_{j}^{t} \frac{\left[W^{T} x\right]_{j}^{+}+\eta 1_{k}}{\left[\left(W^{T} W+\eta 1_{k} 1_{k}^{T}\right) h^{t}\right]_{j}+\left[W^{T} x\right]_{j}^{-}}
\end{aligned}
$$

So far we have proved that the above updating rule can make $E\left(h^{t}\right)$ nonincreasing w.r.t to the $t$. Specifically, we have the lower bound for every element on the diagonal of $J$ matrix.

$$
J\left(h^{t}\right)_{j j}=\frac{\left[\left(W^{T} W+\eta 1_{K} 1_{K}^{T}\right) h^{t}\right]_{j}+\left[W^{T} x\right]_{j}^{-}}{h_{j}^{t}} \geq \eta
$$

Based on 31 and 40 , we can derive the lower bound of the difference between two lose function values after 
one update.

$$
\begin{aligned}
E\left(h^{t}\right)-E\left(h^{t+1}\right) & \geq F\left(h^{t}, h^{t}\right)-F\left(h^{t+1}, h^{t}\right) \\
& =\left(h^{t+1}-h^{t}\right)^{T} \nabla F\left(h^{t+1}, h^{t}\right)+\frac{1}{2}\left(h^{t}-h^{t+1}\right)^{T} \nabla^{2} F\left(h^{t+1}, h^{t}\right)\left(h^{t}-h^{t+1}\right) \\
& =\frac{1}{2}\left(h^{t}-h^{t+1}\right)^{T} J\left(h^{t}\right)\left(h^{t}-h^{t+1}\right) \\
& \geq \frac{\eta}{2}\left\|h^{t}-h^{t+1}\right\|^{2}
\end{aligned}
$$

since $\nabla F\left(h^{t+1}, h^{t}\right)=0$ and $\nabla^{2} F\left(h^{t+1}, h^{t}\right)=J\left(h^{t}\right)$. And by accumulating $\left\|h^{t}-h^{t+1}\right\|^{2}$ from 0 to $T-1$, we have

$$
E\left(h^{0}\right)-E\left(h^{K}\right) \geq \frac{\eta}{2} \Sigma_{t=0}^{T-1}\left\|h^{t}-h^{t+1}\right\|^{2}
$$

Thus, for any given fixed iteration number $T$, we have

$$
\min _{0 \leq t \leq T}\left\|h^{t}-h^{t+1}\right\|^{2} \leq \frac{2}{\eta(T-1)}\left(E\left(h^{0}\right)-E\left(h^{T}\right)\right) \leq \frac{2}{\eta(T-1)} E\left(h^{0}\right)
$$

\section{Rewriting the Loss Function}

To conduct the parameter analysis and validate the optimization convergence, we rewrote the original loss function to a equivalent formula as following.

$$
\begin{aligned}
\min _{W \geq 0, H \geq 0} L(W, H) & =\frac{1}{2}\|X-W H\|_{F}^{2}-\lambda_{1} \operatorname{tr}\left(W^{T} A S\right)-\lambda_{2} \operatorname{tr}\left(W^{T} A_{0}\right) \\
& +\frac{\mu}{2}\|W\|_{F}^{2}+\frac{\eta}{2}\left\|1_{k}^{T} H-1_{N}^{T}\right\|_{2}^{2} \\
& =\frac{1}{2}\|X-W H\|_{F}^{2}+\frac{\mu}{4} \operatorname{tr}\left(W W^{T}\right)-\lambda_{1} \operatorname{tr}\left(W^{T} A S\right) \\
& +\frac{\mu}{4} \operatorname{tr}\left(W W^{T}\right)-\lambda_{2} \operatorname{tr}\left(W^{T} A_{0}\right)+\frac{\eta}{2}\left\|1_{k}^{T} H-1_{N}^{T}\right\|_{2}^{2} \\
& =\frac{1}{2}\|X-W H\|_{F}^{2}+\frac{\mu}{4} \operatorname{tr}\left(W W^{T}-\frac{4 \lambda_{1}}{\mu}\left(W^{T} A S\right)+\frac{4 \lambda_{1}^{2}}{\mu^{2}}(A S)(A S)^{T}\right) \\
& +\frac{\mu}{4} \operatorname{tr}\left(W W^{T}-\frac{4 \lambda_{1}}{\mu}\left(W^{T} A_{0}\right)+\frac{4 \lambda_{1}^{2}}{\mu^{2}} A_{0} A_{0}^{T}\right)+\frac{\eta}{2}\left\|1_{k}^{T} H-1_{N}^{T}\right\|_{2}^{2}+\text { constant } \\
& =\frac{1}{2}\|X-W H\|_{F}^{2}+\frac{\mu}{4}\left\|W-\frac{2 \lambda_{1}}{\mu}(A S)\right\|_{F}^{2} \\
& +\frac{\mu}{4}\left\|W-\frac{2 \lambda_{2}}{\mu} A_{0}\right\|_{F}^{2}++\frac{\eta}{2}\left\|1_{k}^{T} H-1_{N}^{T}\right\|_{2}^{2}+\mathrm{constant}
\end{aligned}
$$

